\title{
Government Interventions in a Dynamic Market with Adverse Selection*
}

\author{
William Fuchs ${ }^{\dagger} \quad$ Andrzej Skrzypacz
}

April 23, 2015

\begin{abstract}
We study government interventions in a dynamic market with asymmetric information. We show that restricting trading opportunities after an initial round of trade is always optimal. Under a sufficient condition it is optimal to subsidize trades only at time zero while imposing prohibitively high taxes afterwards. If interventions are required to generate a Pareto improvement over laissez-faire then trade is only restricted for a short amount of time. If additional sellers can arrive later, the optimal policy entails asset purchases and price controls. Subsidies can greatly enhance welfare but can be detrimental if provided with delay.
\end{abstract}

\section{Introduction}

During times of financial distress, such as those experienced in 2008 after the demise of Lehman Brothers, asset sales are an important source of funds for financial institutions such as banks and insurance companies. Unfortunately, the big gains from trade between those that are liquidity constrained and those that are not may be difficult to realize due to asymmetric information. As in the classic Akerlof (1970) market for lemons, if buyers

*This paper is closely related to our previous working paper: "Costs and Benefits of Dynamic Trading in a Lemons Market."

${ }^{\dagger}$ Fuchs: Haas School of Business, University of California Berkeley (e-mail: wfuchs@haas.berkeley.edu). Skrzypacz: Graduate School of Business, Stanford University (e-mail: skrz@stanford.edu). We thank Ilan Kremer, Mikhail Panov, Christine Parlour, Aniko Öry, Brett Green, Marina Halac, Johanna He, Hugo Hopenhayn, Alessandro Pavan, Jean Tirole, Felipe Varas, Robert Wilson, Pavel Zryumov, and participants of seminars and conferences for comments and suggestions. We also gratefully acknowledge support from the National Science Foundation grant \#1260853. 
were to pay the price corresponding to the average quality of the assets in the market, sellers holding the best assets might not wish to trade. Realizing this, buyers would then reduce their offers and end up trading with a small fraction of the sellers or none at all. Absent government intervention, trade either completely stops or slows down. In the latter case, over time prices gradually rise as better and better assets are traded in the market.

The main questions we seek to answer in this paper are whether and how should the government intervene in these situations, even if it has a binding budget constraint. We answer them in a model of a dynamic competitive market in which liquidity-constrained sellers have private information about their assets and homogenous, liquidity-abundant buyers compete to buy those assets.

Several recent papers document how different financial markets had drastic reductions in volume in the aftermath of Lehman Brothers collapse in 2008. Among others, Heider, Hoerova \& Holthausen (2009) discuss the collapse of the interbank market, McCabe (2010) discusses the money market funds and Duffie (2010) discusses the OTC and repo markets. These contractions were largely driven by the uncertainty over the counterparty's ability to meet its obligations and the disagreement over the value of securities that could be used as collateral. This was clearly reflected in the OTC market where the types of securities acceptable as collateral significantly changed. Information sensitive securities were largely replaced by cash. Similarly, the assets under management of money market funds saw a big compositional change at the time of Lehman's collapse with a pronounced drop in the amount of asset-backed commercial paper and a large increase of government securities. These recent events motivate our interest in dynamic markets with asymmetric information and the impact of government interventions on such markets.

We first characterize the laissez-faire equilibrium (Proposition 1). Assuming a continuum of seller types, competitive buyers, and continuous time, leads to a very tractable equilibrium. It is characterized by a smooth flow of trade where worst assets are sold first and both the quality of traded assets and price gradually increase over time.

Our first policy result (Lemma 1) is that introducing high taxes for an interval of time, $\Delta>0$, after an initial round of potentially subsidized trade, is always a part of an optimal policy. By taxing future trades, the government creates more incentives to trade in the early tax-exempt period. In particular, holders of higher quality assets that would delay trade absent the government policy now prefer to trade earlier in order to avoid the taxes or excessive delay. As the quality of the pool of assets sold early improves, market price increases as well. Higher prices in turn induce even more trade creating a virtuous cycle.

Our second policy result (Theorem 1) shows that under a sufficient regularity condition on 
the shape of the gains from trade and the distribution of asset values, it is optimal to allow only one (potentially subsidized) round of trade at time zero while imposing prohibitively high taxes afterwards (i.e. setting $\Delta=\infty$ ). Intuitively, the regularity condition implies that the ratio of the marginal gains from trade to the marginal information rents of the sellers is decreasing in the asset quality. Under this condition, the solution to the optimization problem has a bang-bang property: it is optimal to push as much trade as possible to take place immediately even if it comes at the expense of excluding all higher types from trade altogether. $^{1}$

Since the optimal policy effectively excludes higher types from trading, these types are worse off than they would be in the laissez-faire equilibrium. An important question and our most novel results come from analyzing optimal policy subject to making all players better off than in the laissez-faire benchmark. We show than in this case it is still always optimal to effectively close the market for some time after the initial round of trade. Under a similar regularity condition to that of Theorem 1, in Theorem 2 we show that the optimal intervention in this case involves an initial round of trade followed by a short period of high taxes, followed again by trading with no taxes. Under the optimal policy, low-quality assets (below a threshold) are pooled and trade at time 0; better assets (above the threshold) are screened perfectly and trade at the same times and terms as in the laissez-faire economy.

In Section 5.1 we extend the model by allowing for additional distressed sellers to arrive over time. The optimal policy in this case does not shut down the market (Theorem 3). Instead, the optimal policy entails asset purchases by the government and price controls. It can be decentralized by controlling market prices: by allowing for just one constant price in the market, the buyers effectively face a take-it-or-leave-it offer whenever they arrive. As in Theorem 1, buyers either trade immediately upon arrival or never trade. Again, they key for efficiency gains is that the policy removes benefits from delaying trade (the terms of trade do not get better), and that reduces the adverse selection problem because higher types pool with lower types.

Throughout the paper we allow the government to carry out non-budget neutral interventions. This is important for both normative and positive considerations. We show that there can be big returns from spending some resources on subsidies/bail-outs in these markets: even if raising a dollar in revenues from another market induces some deadweight loss, it

\footnotetext{
${ }^{1}$ This is related to the result that a durable-good monopolist facing a demand curve with decreasing marginal revenue, would like to commit to set constant price. Such price induces high valuation buyers to buy immediately and the rest of the buyers never to trade, that is, under commitment the seller would not want to use time to screen buyers.
} 
may still be optimal to subsidize these markets. This is best illustrated by showing that in certain cases even with the best budget-neutral intervention there is no trade in the market (see Section 4.4). By providing an initial subsidy the government is able to jump-start the market and greatly increase the overall surplus.

Our results in Section 5.3 stress the importance of acting quickly. Via a series of examples we demonstrate that the timing of the subsidies is crucial. If the government moves slowly and the subsidy is expected to arrive in the future (either deterministically or stochastically) then it can actually have a negative effect on welfare. The intuition is that the expectation of future subsidies delays current trade. So, although there is clearly a benefit from subsidizing trades, it is of the essence that the government acts fast. While the flexibility of the Federal Reserve and its ability to act fast was likely crucial in the recent crisis, some of the uncertainty over future interventions/subsidies may have contributed to the reduction in trade volume in private markets.

Lastly, we note that the exact form of the subsidy is not important, as long as the information rents collected by the different seller types are unchanged (and transaction costs are the same). A proportional subsidy, as assumed for concreteness in the paper, or a government guarantee on the payoff of the assets, as implemented during the crisis with the Public-Private Investment Program for Legacy Assets, would have equivalent effects. Outright purchases of assets, as implemented with the TARP program, would also be equivalent, but only if the program budgeting accounted for the expected future proceeds from the assets purchased by the government. For example, a subsidy program with a budget $b$ is equivalent to a purchase program with a budget $b$, if the budget in the latter case is on net losses from the program and not on total purchases. ${ }^{2}$

\section{Related Literature}

Optimal government interventions in similar models have been studied recently by Philippon and Skreta (2012) and Tirole (2012). In these papers, the government offers financing to firms having an investment opportunity and it is secured by assets that the firms have private information about (these are sellers in our model - using an asset as a collateral or selling it to obtain financing are essentially economically equivalent). That round of government financing is followed by a static competitive market in which firms that did not receive

\footnotetext{
${ }^{2}$ That assumes that the government holding the assets to maturity is as efficient as private buyers holding the asset. If not, then after purchasing the assets the government should pool them into a portfolio and sell shares of the portfolio to buyers with liquidity. Since the government can commit to pool all of the assets, there would be no (additional) adverse selection problem in creation and sales of the portfolio.
} 
funds from the government can raise funds in a private market. This creates a problem of "mechanism design with a competitive fringe" as named by Philippon and Skreta (2012): the government intervention affects the post-intervention equilibrium and vice versa (a feature shared by our model). In sharp contrast to our results, both papers show that tampering with the private markets does not improve welfare: see Proposition 2 in Tirole (2012) and Theorem 2 in Philippon and Skreta (2012). Since the post-intervention market creates endogenous IR constraints for the agents participating in the government program, making the market less attractive could make it easier for the government to intervene. However, these two papers argue that this is never a good idea.

The key difference between our model and these two papers that leads to these opposing results is that Philippon and Skreta (2012) and Tirole (2012) assume a static model of the private market, while we study a dynamic market. It is best seen in the light of our Theorem 1: under the regularity condition, it is indeed optimal to have a government subsidy at time zero and all trade happening at time zero, with no additional trades in the future. Beyond this crucial difference in results and their practical interpretation, our paper differs from these two papers in terms of the focus on the dynamics of trade and the tradeoffs in dynamic interventions.

Lemma 1 and the bang-bang property of the optimal intervention in Theorem 1 are mathematically related to the findings of Samuelson (1984). In a static setting he shows that the optimal budget-neutral mechanism divides sellers into at most three groups: a group that trades with probably one, a group that trades with a common intermediate probability and a group that does not trade. In our dynamic setting this translates respectively to a group that trades immediately, a group that trades with delay and a group that never trades. Our Theorem 1 contributes to his result by establishing a sufficient condition for the optimal mechanism having trade only at $t=0$. Moreover, we show how the optimal direct revelation mechanism can be implemented in a decentralized market with a particular government policy that induces a unique competitive equilibrium. In addition, we also extend the prior results by allowing for non budget-neutral interventions.

Our result on Pareto improving interventions has a historical connection to the political economy models of reform with consensus or majority voting. ${ }^{3}$ Within this literature the closest paper is Brusco and Hopenhayn (2007). They look at the problem of eliminating an inefficient regulation such as trade barrier that keeps unproductive firms in business. Firms are privately informed about their production costs and the optimal policy determines when

\footnotetext{
${ }^{3}$ See for example Fernandez and Rodrik (1989).
} 
such firms must be shut down. One difference with our model is that we have interdependent values between sellers and buyers while in theirs the value to the consumers is independent of the production cost. ${ }^{4}$ Their main result with commitment has firms exiting at most at two times. While this is similar to our Lemma 1, the right comparison is to Theorem 2, where we require the policy to achieve a Pareto improvement. In this case, we have that a subset of types trade at time zero but then all other types perfectly separate from each other. Thus our results, although related, are quite different.

Two related papers, Heider, Hoerova and Holthausen (2009) and Bolton, Santos and Scheinkman (2011), combine the problem of adverse selection with one of maturity mismatch. Although we do not model the maturity mismatch problem explicitly, we believe it had an important role in the recent crisis and our liquidity-constrained sellers likely are in that situation because of it. That is, we see the maturity mismatch problem as a possible micro-foundation of our model of gains from trade and asymmetric information. Heider, Hoerova \& Holthausen (2009) have a 3 period model of the interbank market. Banks in need of liquidity can use the interbank market to borrow from those with excess liquidity. Asymmetric information about the quality of the assets in the borrower's balance sheets makes lenders afraid of lending to a "lemon" leading to a reduction or complete disappearance of credit. They discuss some policy interventions but their focus is positive rather than normative and essentially static. ${ }^{5}$

On the theoretical side, our paper is also related to literature on dynamic markets with adverse selection. The closest paper is Janssen and Roy (2002) who study competitive equilibria in a market that opens at a fixed frequency. They show that in equilibrium prices increase over time and eventually every type trades. They do not ask market design or policy questions as we do in this paper. Yet, we share with their model the observation that dynamic trading leads to increasingly better types trading over time. Camargo and Lester (2014) find the same equilibrium dynamics in a setting with decentralized search rather than a competitive market (in discrete time, with two types of the seller). While their paper is focused on characterizing the set of equilibria of the game with no government intervention, they also show that sunset provisions can increase benefits of government subsidies. Sunset provisions are useful because, as we show in this paper, expectation of future subsidies can slow down current trade. Our paper shows other examples of problems of delayed/prolonged

\footnotetext{
${ }^{4}$ They also have heterogeneous consumers that obtain consumer surplus, while we have homogenous buyers that make zero profits in equilibrium.

${ }^{5}$ Bolton, Santos and Scheinkman (2011) is a bit further from our work since they focus more on the ex-ante asset choices and assume there is no asymmetric information initially but that it grows over time.
} 
interventions and adds to this analysis by characterizing the optimal polices. Also in a search setting with two types of asset Chiu and Koeppl (2011) argue that search frictions are crucial for adverse selection to play an important role and that delaying the asset purchasing programs might be a good idea. Our model has no search frictions yet adverse selection can cause the market to completely shut down and we show that delaying interventions is costly.

On the more technical side, our competitive-equilibrium setup with a continuum of types and continuous time allows us to show uniqueness of equilibrium, which makes it easier to interpret comparative statics. For other papers on dynamic signaling/screening with a competitive market see Noldeke and van Damme (1990), Swinkels (1992), Kremer and Skrzypacz (2007) and Daley and Green (2012). While we share with these papers an interest in dynamic markets with asymmetric information, none of these papers studies government interventions.

While in this paper we study government interventions in terms of taxes and subsidies, there are other ways the government or market designer can affect trade in equilibrium. For example, in our related working paper, Fuchs and Skrzypacz (2013), we allow the market designer to determine the times the market should be open or closed. The market microstructure literature (see Biais, Glosten and Spatt (2005)) has also considered the question of how different trading protocols perform in the presence of adverse selection. That literature has mainly focused on the stock markets where there are potentially many competing sellers, divisible assets and dispersed information. A different design question for dynamic markets with asymmetric information is asked in Hörner and Vieille (2009), Kaya and Liu (2012), Kim (2012) and Fuchs, Öry and Skrzypacz (2015). These papers ask how information about past rejected offers affects efficiency of trade. Moreno and Wooders (2012) ask a yet another design question: they compare decentralized search markets with centralized competitive markets.

\section{The Model}

There is a mass of size one of financially distressed banks (the sellers). Each seller owns one unit of an indivisible asset. When the seller holds the asset, it generates for him a revenue stream with net present value $c \in[0,1], x$ that is private information of the seller. The seller types, $c$, are distributed according to $F(c)$, which is common knowledge, atomless and has a continuous, strictly positive density $f(c)$. We assume that the private information is never 
revealed. ${ }^{6}$

There is a competitive market of potential buyers. Each buyer values the asset at $v(c)$ which is strictly increasing, twice continuously differentiable, and satisfies $v(c)>c$ for all $c \in(0,1), v(0) \geq 0$, and $v(1)=1$ (i.e. no gap on the top) ${ }^{7}$

Time is $t \in[0, \infty]$ and the market is continuously open. There is also a benevolent government that can intervene by subsidizing or taxing trades proportionally. ${ }^{8,9}$ The government publicly commits to a path of taxes $\tau_{t}$ for $t \in[0, \infty]$ before the market opens at $t=0$. If at time $t$ buyers pay price $p_{t}$, the sellers receive $p_{t}\left(1-\tau_{t}\right) ; \tau_{t}<0$ represents a subsidy.

All players discount payoffs at a rate $r$. If a seller with type $c$ sells at time $t$ at a price $p_{t}$, its payoff is

$$
\left(1-e^{-r t}\right) c+e^{-r t} p_{t}\left(1-\tau_{t}\right)
$$

and the buyer's payoff at the time of purchase is:

$$
v(c)-p_{t}
$$

Given a path of prices and taxes, the sellers face an optimal stopping problem. Namely, when to sell and collect $p_{t}\left(1-\tau_{t}\right)$ :

$$
\max _{\hat{t}}\left(1-e^{-r \hat{t}}\right) c+e^{-r \hat{t}} p_{\hat{t}}\left(1-\tau_{\hat{t}}\right)
$$

Since the stopping problem is supermodular in $c$ and $\hat{t}$, if seller of type $c$ has an optimal stopping time $t$ then all types $c^{\prime}<c$ have optimal stopping times $t^{\prime} \leq t$ (even if the optimal stopping time for some types is not unique). The intuition is that the lower types get the same payoff from selling as type $c$, but forego less of future cash flows. This is known as the "skimming property" and it simplifies equilibrium analysis since in equilibrium the set of seller types remaining in the market at any time is a truncation of the original seller distribution.

\footnotetext{
${ }^{6}$ Most of our results can be extended to a setting in which at some deterministic or random time the private information becomes public, but the players cannot contract on the realization of this information (see the working paper Fuchs and Skrzypacz 2013). While the cashflows generated by the asset (which are correlated with $c$ ) are realized by the buyer, we assume that the buyer and the seller cannot contract on their realization, i.e., we assume that the seller cannot offer warranty contracts on the assets he sells.

${ }^{7}$ Assuming $v(1)=1$ allows us not to worry about out-of-equilibrium beliefs after a history where all sellers were supposed to trade but some did not trade. The equilibria we characterize in this paper continue to exist even if $v(1)>1$ but may no longer be unique. A monotonicity condition on beliefs would select this equilibrium.

${ }^{8}$ As discussed in Remark 1, the exact form of the subsidy turns out not to matter.

${ }^{9}$ In Section 5 we allow for a richer set of instruments.
} 
Let $\hat{t}^{*}(c)$ be some selection of the optimal stopping times given the net-price process, $p_{t}\left(1-\tau_{t}\right) \cdot{ }^{10}$ Let $k_{t}$ denote the lowest quality asset that has not been traded by time $t$ :

$$
k_{t}=\inf \left\{c: \hat{t}^{*}(c) \geq t\right\}
$$

Note that $k_{t}$ is left-continuous and it is independent of the selection of the optimal stopping times (since for any $t$ at most zero measure of types are indifferent between stopping at that time and some other time).

We use $K_{t}$ to describe the (set of) types that trade at $t$. There are three possibilities: (i) if $k_{t}$ is constant to the right of $t$, that means there is no trade at $t$, and we denote it by $K_{t}=\emptyset$; (ii) if $k_{t}$ increases continuously to the right of $t$, it means trade is smooth at $t$, and we denote it by $K_{t}=k_{t}$; (iii) if $k_{t}$ jumps discontinuously from $k_{t}$ to $k_{t^{+}}=\lim _{s \rightarrow t^{+}} k_{s}$, it means that there is an atom of trade at $t$, and we denote by $K_{t}=\left[k_{t}, k_{t^{+}}\right]{ }^{11}$

With this notation we define a competitive equilibrium:

Definition 1 Given a tax schedule $\left\{\tau_{t}\right\}$, a competitive equilibrium is a pair of functions $\left\{p_{t}, k_{t}\right\}$ for $t \geq 0$ that satisfy:

(E1) Zero Profit Condition: if $K_{t} \neq \emptyset$, then $p_{t}=E\left[v(c) \mid c \in K_{t}\right]$

(E2) Seller Optimality: Sellers optimally choose their stopping times (i.e., $k_{t}$ is consistent with seller optimization given $p_{t}$ and $\left.\tau_{t}\right)$.

(E3) Market Clearing: for all $t, p_{t} \geq v\left(k_{t}\right)$.

Conditions $(E 1)$ and $(E 2)$ are standard. Condition (E3) deserves a bit of explanation. It is needed because condition $(E 1)$ provides no discipline when $K_{t}=\emptyset$. We justify it by a market clearing reasoning, that is, that given the market prices, demand equals supply. Suppose at some $t$ the assets were offered at $p_{t}<v\left(k_{t}\right)$. Then, since all buyers believe that the value of the assets is at least $v\left(k_{t}\right)$, they would all demand it. Demand would not equal supply and the market would not clear. ${ }^{12}$ This condition removes some trivial multiplicity of equilibria. For example, it removes as a candidate equilibrium the path $\left(p_{t}, k_{t}\right)=(0,0)$ for all periods (i.e. no trade and very low prices) even though this path satisfies the first two conditions. $^{13}$

\footnotetext{
${ }^{10}$ To assure the stopping problem has a solution, we restrict $\tau_{t}$ to be such that we can construct equilibrium $p_{t}$ so that $p_{t}\left(1-\tau_{t}\right)$ is right-continuous when it is increasing and left-continuous when it is decreasing.

${ }^{11}$ We use the notation $k_{t^{+}}, p_{t^{+}}$, etc., to denote right-limits of the corresponding functions at $t$.

${ }^{12}$ We thank Andrew Postlewaite for pointing this out to us.

${ }^{13}$ Condition (E3) is analogous to the condition (iv) in Janssen and Roy (2002) and is weaker than the No Unrealized Deals condition in Daley and Green (2012) (see Definition 2.1 there; since they study the gap case with private offers, they need a stronger condition to account for out-of-equilibrium beliefs).
} 
We assume that all market participants publicly observe all the trades. Hence, once a buyer purchases an asset, if he tries to put it back on the market, the market makes a correct inference about $c$ based on the history. Since we assume that all buyers have the same value of the asset, there would not be any profitable re-trading of the asset (after the initial seller transacts) and, hence, we ignore that possibility.

\subsection{Laissez-faire Equilibrium}

Absent government interventions, i.e., if $\tau_{t}=0$ for all $t$, the equilibrium has no atoms of trade and is given by: ${ }^{14}$

Proposition 1 (laissez-faire) If $\tau_{t}=0$ for all $t$, there exists a unique competitive equilibrium. The equilibrium is the unique solution to:

$$
\begin{aligned}
p_{t} & =v\left(k_{t}\right) \\
k_{0} & =0 \\
r\left(v\left(k_{t}\right)-k_{t}\right) & =v^{\prime}\left(k_{t}\right) \dot{k}_{t} .
\end{aligned}
$$

To see the intuition (proof is in the appendix), note that if an interval of types traded at time $t$, Condition (E3) would require that an instant later $p_{t^{+}} \geq v\left(k_{t^{+}}\right)$. However, that would imply a jump in prices at $t$ since $p_{t}=E\left[v(c) \mid c \in K_{t}\right]<v\left(k_{t^{+}}\right)$. But if prices jumped, no type would trade the instant before the jump. Hence, $k_{t}$ is a continuous function in equilibrium. Therefore, by Condition $(E 1), p_{t}=v\left(k_{t}\right)$ when there is trade. The differential equation for $k_{t}$ comes from seller optimality: at each point in time, the current cutoff type $k_{t}$ must be indifferent between trading or delaying trade for an instant. The gain from waiting for an instant of time is that prices rise over time while the cost of delaying trade is the lost interest on the gains from trade. Together:

$$
r\left(p_{t}-k_{t}\right)=\dot{p}_{t}
$$

Using $p_{t}=v\left(k_{t}\right)$, this tradeoff can be stated as:

$$
r\left(v\left(k_{t}\right)-k_{t}\right)=v^{\prime}\left(k_{t}\right) \dot{k}_{t}
$$

\footnotetext{
${ }^{14} \mathrm{By} \dot{k}_{t}$ we denote the time derivative of $k_{t}$.
} 
This differential equation, together with the boundary condition $k_{0}=0$, pins down the equilibrium path of $k_{t}$.

We obtain this remarkably simple characterization of the equilibrium in a dynamic market with adverse selection thanks to a combination of assumptions: continuous time, continuum of types, and frictionless, competitive-buyers market. Without it, there could be multiple equilibria, they would not be fully separating, and would be typically hard to characterize. For example, if time is discrete, in every period an atom of types would trade and there would be in general a multiplicity of equilibria (as in Janssen and Roy 2002).

Note that if $v(0)=0$ (i.e. no strict gains from trade in the bottom of the distribution), there is no trade in equilibrium. ${ }^{15}$ Moreover, the dynamics of $\left(p_{t}, k_{t}\right)$ in the laissez-faire equilibrium do not depend on the shape of the distribution $F(c)$ but only on its support. The shape of $F(c)$ will play a role once we introduce government interventions that generate atoms of trade. Finally, total surplus/gains from trade in the laissez-faire equilibrium are:

$$
S_{L F}=\int_{0}^{\infty} e^{-r t}\left(v\left(k_{t}\right)-k_{t}\right) \dot{k}_{t} f\left(k_{t}\right) d t=\int_{0}^{1} e^{-r \tilde{t}(c)}(v(c)-c) f(c) d c
$$

where $\tilde{t}(c)$ is the inverse of $k_{t}$. Condition (3) implies $\tilde{t}^{\prime}(c)=\frac{v^{\prime}(c)}{r(v(c)-c)}$ and hence $S_{L F}$ is independent of the discount factor (since $c$ and $v(c)$ are present values, the first-best surplus is independent of $r$ as well). The intuition is that since in equilibrium all types eventually trade, the deadweight loss is due to the delay of trade. While a smaller discount implies that any fixed delay is less costly, by condition (3) a smaller $r$ implies more delay. Since the speed of trading, $\dot{k}_{t}$, is proportional to $r$, these two forces cancel each other out.

\section{Government Interventions: Motivating Examples}

We start with the following benchmark example to illustrate the benefits of imposing future taxes to increase early trading. Assume $c$ is distributed uniformly over $[0,1]$ and $v(c)=\frac{1+c}{2}$, as illustrated in Figure 1.

\footnotetext{
${ }^{15}$ This can be seen from equation (3). Continuous trading is not necessary for this result to hold. If trading is at discrete intervals of time $\Delta$ this result would continue to hold for small $\Delta$ if $f(0)>0$. Even for $\Delta=\infty$ the result may continue to hold, as we show in Section 4.4.
} 


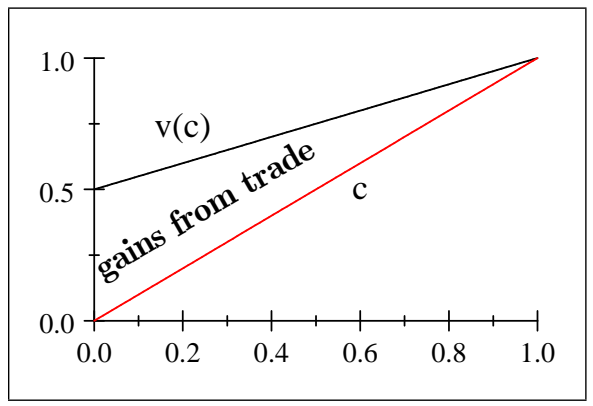

Figure 1: Example of gains

from trade.

\section{Laissez-faire Economy}

Absent any government interventions in this example the equilibrium cutoffs are:

$$
k_{t}^{L F}=1-e^{-r t}
$$

The total surplus in the laissez-faire economy is:

$$
S_{L F}=\int_{0}^{1} e^{-r \tilde{t}(c)}(v(c)-c) f(c) d c=\frac{1}{6} .
$$

Note that even though asymptotically all types trade in equilibrium, the equilibrium is inefficient due to delay. The first-best has all types trading immediately (i.e., $\tilde{t}(c)=0)$ and surplus is $\int_{0}^{1}(v(c)-c) f(c) d c=\frac{1}{4}>S_{L F}$.

\section{Initial Subsidy Followed by Constant Permanent Tax}

Now consider the following government intervention. The government provides an initial subsidy $s=-\tau_{0} \geq 0$ per unit traded at time 0 and then finances this subsidy with a constant tax rate $\tau_{t}=\tau$ for $t>0$, to (dynamically) achieve budget balance. One interpretation of this intervention is that the government levies a constant tax rate on the market to finance a subsidized auction at time 0 .

To construct the equilibrium we solve the following fixed point problem. We first solve for the equilibrium for any $s$ and $\tau$. Then, we look for pairs of $(s, \tau)$ that are budget neutral. The amount of initial trade depends on the initial subsidy; how much of a subsidy can be provided depends on the amount of trade after $t>0$ which, in turn, is a function of which types trade at $t=0$. 
The one-time subsidy at time 0 induces an atom of trade at time 0 and the constant tax later implies either no trade (if the tax is high enough) or smooth trade. We provide the detailed computations in the Appendix and focus here on how the dynamics and welfare depend on $\tau$.

How does the equilibrium depend on $\tau$ ?

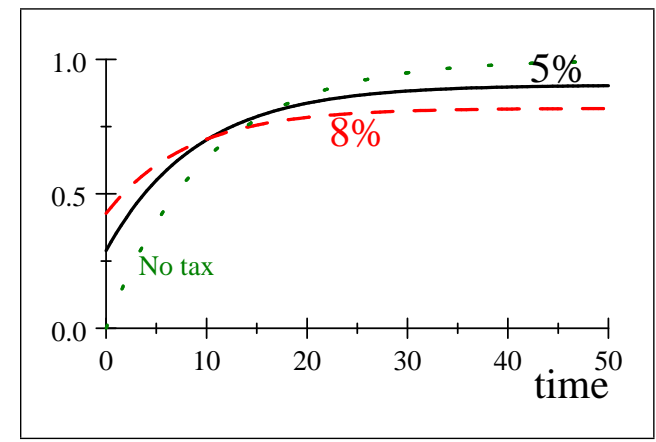

Figure 2: Taxes and Cutoff

Dynamics

In Figure 2 we plot equilibrium cutoffs, $k_{t}$, for different tax rates: the dashed line has $\tau=15 \%$, the solid line $\tau=5 \%$ and the dotted line has $\tau=0 \%$. As shown, a higher tax rate leads to a higher initial cutoff but slower trade thereafter. In other words, as taxes increase, there is a tradeoff between trading faster with the lower types at the expense of slower trade with higher types.

How does the total surplus change with $\tau$ ?

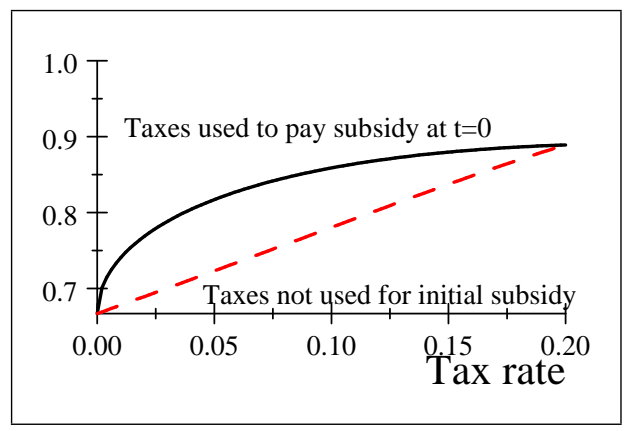

Figure 3: Surplus relative to first

best with a tax-exempt auction

followed by a constant tax. 
Figure 3 shows that in this example surplus (the solid line, represented as a fraction of first-best surplus) monotonically increases in the level of taxes. For sufficiently high taxes $(\tau \geq 20 \%)$, there is no trade after $t=0$ and the surplus is only $11 \%$ below first best. With no intervention it is $33 \%$ below.

Somewhat surprisingly, even if the taxes are levied but not used for the initial subsidy (i.e., if $s=0$ but $\tau>0$ ), the surplus is also increasing in $\tau$, as shown by the dashed line in Figure 3. Comparing the two curves, for small tax rates, the initial subsidy has a large contribution to the welfare gains; yet, for large tax rates the difference is small. The reason is that for high tax rates the Laffer Curve implies that total tax revenues decrease in the tax rate and hence the subsidy becomes again small. The main effect of taxes is then that they push more sellers to participate in the initial tax-exempt auction, and that improves efficiency.

\section{Optimal Government Interventions}

In this section we return to our general model and provide three policy results. We assume that the government has a fixed intertemporal budget (see equation (5) for a formal statement of the constraint) and chooses a policy to maximize total surplus subject to the budget constraint. First, for a general distribution of sellers' valuations, $F(c)$, and buyers' values, $v(c)$, we show that an optimal policy always includes high taxes for some time $\Delta$ after time $t=0$. This effectively closes the market for $t \in(0, \Delta)$ and induces bunching of trades at time $t=0$. Importantly, as more seller types sell at time $t=0$ because of the taxes soon after, the equilibrium price increases, inducing even more trade. Second, under a regularity condition on $F(c)$ and $v(c)$, we characterize optimal interventions for a given budget constraint. It involves using all the resources to subsidize trade at $t=0$ and setting $\Delta=\infty$, effectively allowing only one opportunity to trade. Third, we characterize an optimal policy subject to guaranteeing that the policy makes all types better off than under laissez-faire..

\subsection{Partial Market Closure is Always Optimal}

The following result captures the central idea of this paper. Restricting the opportunities to trade for some times is always part of optimal policy.

Lemma 1 Suppose $v(0)>0$. For every $r, F(c)$, and $v(c)$, there exists $\Delta>0$ such that an optimal policy has sufficiently high taxes for $t \in(0, \Delta)$, so that there is no trade during that time. 
We provide in the appendix a combined proof of Lemma 1 and Theorem 1, and explain the intuition for both results after Theorem 1 . To prove Lemma 1 we show that an optimal policy results in trade in at most two distinct times and use mechanism design tools to establish the claim. ${ }^{16}$

In Lemma 1, we assume that $v(0)>0$. In case $v(0)=0$, there is no trade in the laissezfaire equilibrium. Therefore, if taxes could induce any trade, we would get a strict welfare improvement. It is always possible if the government has a positive budget to provide a net subsidy. Whether it is possible even with no subsidies depends on the shapes of $F(c)$ and $v(c)$. When the government cannot use subsidies, in some cases, even the optimal policy cannot induce any trade (for an example see Section 4.4); while in some cases taxes alone can induce trade and improve welfare (for example, when $F(c)=c$ and $v(c)=\sqrt{c}$ ).

\subsection{When Extreme Policy is Optimal}

Lemma 1 shows that closing the market for a potentially short amount of time, $\Delta$, is always part of an optimal policy. We next show that under a regularity condition the optimal policy calls for $\Delta=\infty$. That is, effectively allowing trade only once.

Definition 2 We say that the environment is (strictly) regular if $\frac{f(c)}{F(c)}(v(c)-c)$ is (strictly) decreasing.

The ratio $\frac{f(c)}{F(c)}(v(c)-c)$ represents the relative marginal effect of speeding up trade of type $c$ on the social surplus and the information rent of the seller. This regularity condition is also similar to the standard condition in price theory that the marginal revenue is monotone. In particular, think about a static problem of a monopsonist buyer choosing volume of trade, $F(c)$, by making a take-it-or-leave-it offer equal to $P(c)=c$. The F.O.C. of this problem is: $f(c)(v(c)-c)-F(c)=0$. A decreasing ratio $\frac{f(c)}{F(c)}(v(c)-c)$ guarantees that the marginal profit (the left-hand-side of the F.O.C.) crosses zero exactly once. If we assumed constant gains from trade, as in Philippon and Skreta (2012), then the regularity condition would have been simply a monotone hazard rate property (i.e. log-concavity of the distribution of types), a standard assumption in mechanism design. Under this condition we can prove the following result.

\footnotetext{
${ }^{16}$ After a transformation of the problem, the proof is mathematically analogous to the proof of Lemma 1 in Samuelson (1984)
} 
Theorem 1 If the environment is regular, a competitive equilibrium for a tax policy

$$
\tau_{t}=\left\{\begin{array}{c}
s \leq 0 \text { for } t=0 \\
\tau \geq H \text { otherwise }
\end{array},\right.
$$

where the initial subsidy s consumes all the government's budget and $H$ is high enough so that there is no trade after $t=0$, maximizes total surplus over all possible policies and all corresponding equilibria.

Moreover, if the environment is strictly regular, the competitive equilibrium for this tax policy is unique.

The details of the proof are in the appendix, but here we outline the main steps (and provide intuition for Lemma 1 as well).

We start with a mechanism design problem. A market designer maximizes expected gains from trade by choosing a direct revelation mechanism (mapping reported types into a probability distribution over times of trade and into payments), is allowed to cross-subsidize sellers trading in different periods, but has to satisfy the budget constraint on average. We then argue that the optimal mechanism can be implemented by a tax policy and a corresponding equilibrium.

For a given mechanism, let $G_{t}(c)$ denote, for a given type, the (cumulative) distribution over times of trade. The expected discounted time to trade for this type is then:

$$
x(c)=\int_{0}^{\infty} e^{-r t} d G_{t}(c)
$$

and since all the traders are risk-neutral, their expected payoffs and total surplus depend on $G_{t}(c)$ only via $x(c)$.

In the direct revelation mechanism, the designer chooses $x(c)$ and a net transfer to type c, $P(c)$, to maximize overall welfare:

$$
\max _{x(c), P(c)} \int_{0}^{1} x(c)(v(c)-c) f(c) d c
$$

subject to the budget constraint:

$$
\int_{0}^{1}[x(c) v(c)-P(c)] f(c) d c+b \geq 0
$$


where $b \geq 0$ is the government's budget; ${ }^{17}$ the truth-telling constraint:

$$
c \in \arg \max _{\tilde{c}}(1-x(\tilde{c})) c+P(\tilde{c})
$$

and individual rationality for the seller.

Truth-telling implies that the equilibrium payoff of type $c, U(c)$, has to satisfy $U^{\prime}(c)=$ $(1-x(c))$ almost everywhere, and $x(c)$ has to be weakly decreasing. As usual, in any optimal mechanism the highest type, $c=1$, is kept down to its outside option, $U(1)=1$. We use this to combine the truth-telling and the budget constraints and express the latter in terms of the allocation only, $x(c)$ :

$$
\int_{0}^{1} x(c)\left(v(c)-c-\frac{F(c)}{f(c)}\right) f(c) d c+b \geq 0
$$

In the decentralization of the optimal mechanism we describe below, buyers and sellers trade directly with each other, but in the mechanism design approach we assume the designer acts as an intermediary, committing to a mechanism in which it buys the assets from the sellers and resells them immediately to buyers. Constraint (6) incorporates buyers' IR constraints by assuming that the designer re-sells the assets at a price equal to the expected value of the asset conditional on the set of sellers trading in a given period. In this way, buyers' IR constraints bind, the designer resells efficiently, and $\int_{0}^{1} x(c) v(c) f(c) d c$ is the total expected present value of the revenue the designer collects from buyers. Constraint (6) also incorporates the IR and IC constraints of the sellers: the minimal amount the designer has to pay the sellers is $\int_{0}^{1} x(c)\left(c+\frac{F(c)}{f(c)}\right) f(c) d c$ where $c+\frac{F(c)}{f(c)}$ is the usual virtual cost of trading with type $c$ that combines the IR constraint of each type and the information rent.

Next, the Lagrangian for the problem is linear in $x(c)$, with the derivative of with respect to $x(c)$ :

$$
L(c)=(v(c)-c) f(c)(1+\Lambda)-\Lambda F(c)
$$

where $\Lambda>0$ is the Lagrange multiplier on constraint (6).

Notice first that $v(0)>0$ and $f(0)>0$ imply that $L(0)>0$. Since $L(c)$ is continuous and incentive compatibility requires that $x(c)$ is decreasing, it is optimal to set $x(c)=1$ for $c<c^{*}$, for some $c^{*}>0$. If the budget constraint does not bind, the Lagrange multiplier $\Lambda=0$. Then $L(c)$ is positive for all $c$ and the optimal $c^{*}=1$. In that case all types trade at

\footnotetext{
${ }^{17}$ Budget constraint (5) incorporates the government's option to either hold the assets to maturity or sell them to the buyers at a fair price, in either way receiving $\int_{0}^{1} x(c) v(c) f(c) d c$ in expectation. See discussion below.
} 
time $t=0$ and there is no loss of closing the market after time 0 . When the budget constraint binds, then $L(1)$ is strictly negative. Therefore $L(c)$ crosses zero at least once.

The regularity condition implies that for any $\Lambda>0, L(c)$ crosses zero only once. That, in turn, is a sufficient condition for the optimal solution to have a bang-bang property: types below a threshold $c^{*}$ such that $L\left(c^{*}\right)=0$ trade immediately, and types above the threshold never trade. That solution can be implemented by a competitive equilibrium imposing sufficiently high taxes after the initial trade. Given these taxes, if the regularity condition holds strictly, the equilibrium is unique. Hence this extreme intervention is the most efficient. ${ }^{18}$

What happens when $L(c)$ crosses 0 more than once? Now it may be possible that the optimal $x(c)$ has an intermediate value in $(0,1)$ for $c$ in some range $\left(c_{l}, c_{h}\right)$ (for "ironing" reasons familiar from mechanism design). The claim is that there is always an optimal mechanism in which there is at most one range with such an intermediate $x(c)$. The intuition is as follows. Suppose in the interval $\left(c_{1}, c_{2}\right)$, the optimal $x(c)=\chi_{1} \in(0,1)$, and in the interval $\left(c_{2}, c_{3}\right)$, the optimal $x(c)=\chi_{2} \in\left(0, \chi_{1}\right)$ (the monotonicity of $x(c)$ requires that $\left.\chi_{1} \geq \chi_{2}\right)$. In both of these intervals, the average contribution of these types to the budget constraint, $v(c)-c-\frac{F(c)}{f(c)}$, must be negative since otherwise we could improve upon the mechanism by increasing $x(c)$ (it would improve (4) while relaxing (6)). Now, consider increasing $\chi_{2}$ by $\varepsilon_{2}$ and decreasing $\chi_{1}$ by $\varepsilon_{1}$ in a way that the constraint (6) still binds. If the net effect on (4) is weakly positive, we can do it until $x(c)$ is constant on the range $c \in\left(c_{1}, c_{3}\right)$, and at least weakly improve total surplus. If the net effect is strictly negative, we can strictly improve the objective (4) by reducing $\chi_{2}$ by $\varepsilon_{1}$ and increasing $\chi_{1}$ by $\varepsilon_{1}$. We can do it until either $\chi_{1}=1$ or $\chi_{2}=0$, removing the possibility that the optimal mechanism requires trade in more than two periods. ${ }^{19}$

When the optimal mechanism has trade only at $x \in\{0, \chi, 1\}$, we can implement it via a policy that induces trade only at times 0 and $\Delta$, such that $\chi=e^{-r \Delta}$, and high taxes in any other period so that no type trades in times other than 0 and $\Delta$. Knowing which types are supposed to trade at $\Delta$, we can figure out the market price and subsidy so that the highest type is indifferent between trading at that price and holding the asset forever (and

\footnotetext{
${ }^{18}$ Details of the proof are in the appendix. The proof uses standard mechanism design tools, similar to Samuelson (1984) in a static environment. The contribution of our proof is to apply these methods to characterize optimal intervention in a dynamic market. On the technical side, we contribute by establishing a sufficient condition for the optimal mechanism having trade only at $t=0$, and showing that the described policy induces a unique competitive equilibrium that implements the outcome of the optimal direct revelation mechanism.

${ }^{19}$ Note that the reasoning relies on both the objective function and the constraint being linear in the allocation, $x$.
} 
the subsidy has to be such that the buyers break even on average). For time 0, the tax or subsidy has to be such that the marginal type is indifferent between trading immediately, and waiting to trade at $\Delta$ and the higher price. In case the optimal mechanism has trade at $\Delta$ and $b=0$, the optimal policy requires a tax at time 0 that is used to subsidize trade at time $\Delta$, since as we argued above, the traders at $\Delta$ contribute negatively to the budget constraint.

Policy implications of our Lemma 1 and Theorem 1 are in stark contrast to recent results in the literature. Optimal government interventions in similar models (although, admittedly richer) have been studied recently by Philippon and Skreta (2012) and Tirole (2012). In these papers, the government offers financing to firms having an investment opportunity and it is secured by assets that the firms have private information about. That intervention is followed by a static competitive market in which firms that did not receive funds from the government can trade in a private market to raise funds. This creates a problem of "mechanism design with a competitive fringe" as named by Philippon and Skreta (2012): the government intervention affects the post-intervention equilibrium and vice versa. This effect is shared by our model: in our example from Section 3 the amount of trade at $t=0$ depends on the price after the taxes are removed and that in turn depends on which types trade at time 0 .

Both papers obtain a result that shutting down the private market does not improve welfare: see Proposition 2 in Tirole (2012) and Theorem 2 in Philippon and Skreta (2012). Since the post-intervention market creates endogenous IR constraints for the agents participating in the government program, making the market less attractive could make it easier for the government to intervene. However, these two papers argue that this is never a good idea.

As hinted by our benchmark example and generalized in Lemma 1 and Theorem 1, taking into account the dynamic nature of the market changes this conclusion. The key difference in the models that leads to these opposing results is that both Philippon and Skreta (2012) and Tirole (2012) assume a static model of the private market, while we study a dynamic market. Setting taxes prohibitively high after initial trade, so that all trade takes place at $t=0$ in our model, turns out to be equivalent to assuming that the private market is opened only once after the government intervention, as in their papers. Since their models satisfy the regularity condition, such one-time-trading with no government intervention is optimal by our Theorem 1. However, if the market is dynamic, our results show that some taxes pushing trade to bunch early are optimal. 


\subsection{Pareto Improving Interventions}

It is worth noting that the optimal ex-ante policy generally makes low types better off than in the laissez-faire economy but makes high types worse off. High types eventually trade in the laissez-faire equilibrium but with the optimal intervention they never trade. A natural question to ask then is what is the optimal government intervention that would make all types weakly better off than their laissez-faire outcome. This is a hard problem because the standard mechanism design approach does not work well in this case. These new constraints, which we incorporate as IR constraints, do not allow us to do simple pointwise maximization.

As a first step, it is worth noting that having a short period $t \in(0, \Delta)$ with high taxes can lead to a Pareto improvement over the laissez-faire economy. The high taxes lead to a market closure during the period $\Delta$. The closure generates bunching of trades at $t=0$, which in turn leads to higher prices and even more trade. This positive feedback effect leads to more trade at $t=0$ with the closure than one would get in the time interval $t \in[0, \Delta]$ without the closure. ${ }^{20}$ This is illustrated in the Figure 4 below. For short closures, the solid line representing the cutoff at time zero is above the laissez-faire cutoff (dashed line).

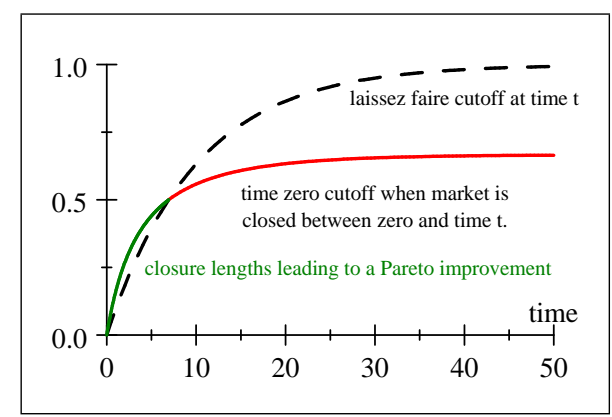

Figure 4: Closures and Trade

Importantly, since after time $\Delta$ there are no more taxes, the differential equation describing the equilibrium path of $k_{t}$ for $t>\Delta$ is the same as in the laissez-faire economy. The only difference in equilibrium comes from the initial condition at time $t=\Delta$ : with a short closure, the cutoff at $t=\Delta$, which we denote by $\kappa_{\Delta}$, is higher than that without the closure, which we denote by $k_{\Delta}^{L F}$. Thus, all types higher than $\kappa_{\Delta}$ trade sooner with the closure and since they trade at the same prices (since these types are fully separated) they are strictly better off than in the laissez-faire economy. To see that lower types are also better off, note that, by revealed preference, type $\kappa_{\Delta}$ is no worse off by pooling since it could wait until $\Delta$ and

\footnotetext{
${ }^{20}$ See the intuition following the formal statement of the result below.
} 
trade at $v\left(\kappa_{\Delta}\right)$. Lastly, recall that in the laissez-faire economy all types $k<\kappa_{\Delta}$ are worse off than type $\kappa_{\Delta}$ because $U(c)$ is increasing. In contrast, with the short closure, types $k<\kappa_{\Delta}$ get the same payoff as type $\kappa_{\Delta}$. Thus they are strictly better off with the intervention than in the laissez-faire economy. Summing up, this policy is a Pareto improvement.

Formally we consider the following tax policy:

$$
\tau^{\Delta} \equiv \tau_{t}=\left\{\begin{array}{rr}
0 \text { for } t \in\{\{0\} \cup[\Delta, \infty)\} \\
\tau>0 & \text { otherwise }
\end{array}\right.
$$

That is, there is tax-exempt trading at $t=0$, followed by a (short) time interval $\Delta$ in which transactions are taxed at a high rare $\tau$ such that no type wants to trade, after which taxes are reduced back to zero.

Lemma 2 Suppose $v(0)>0$. For every $r, F(c)$, and $v(c)$, there exists $\Delta>0$ such that an equilibrium with government policy $\tau^{\Delta}$ yields strictly higher gains from trade than the laissez-faire equilibrium. Moreover, it is preferred by all seller types (Pareto improvement).

The key step of the proof is to show that $k_{\Delta}^{L F}<\kappa_{\Delta}$ for sufficiently low $\Delta$. In turn, to show this, the key result (derived in the Appendix) is that:

$$
\lim _{\Delta \rightarrow 0} \frac{\partial \kappa_{\Delta}}{\partial \Delta}=2 \lim _{\Delta \rightarrow 0} \frac{\partial k_{\Delta}^{L F}}{\partial \Delta}
$$

Since as $\Delta \rightarrow 0$ both $\kappa_{\Delta}$ and $k_{\Delta}^{L F}$ converge to 0 , this means that for small $\Delta$ approximately twice as many types trade before $\Delta$ if the government intervenes in $(0, \Delta)$.

The intuition is as follows. As the government announces the tax plan $\tau^{\Delta}$, some types that were planning to trade in $(0, \Delta)$ now would prefer to trade at 0 even if the price at 0 did not change. The reason is that not taking the price $p_{0}$ implies a fixed delay cost. It turns out that the set of types that decide to take that fixed $p_{0}$ grows in $\Delta$ approximately as fast as does $k_{\Delta}^{L F}$.

The doubling of early trade is then achieved because pooling of trade at time 0 reduces the adverse selection faced by the buyers and hence the price $p_{0}$ increases. For small $\Delta$ the price is approximately half way between $v(0)$ and $v\left(\kappa_{\Delta}\right)$. As the price goes up, even more types prefer to trade at 0 and the adverse selection problem is reduced even further, making $p_{0}$ even higher, and so on. Because prices grow at half the speed of $v\left(k_{\Delta}^{L F}\right)$, the resulting cutoff, $\kappa_{\Delta}$, is twice as high as $k_{\Delta}^{L F}$.

We now consider an optimal policy that is a Pareto improvement over the laissez-faire equilibrium. That is, we ask what mechanism $(x(c), P(c))$ maximizes (4) subject to (5) and 
constraints:

$$
U(c) \geq U_{L F}(c) \text { for all } c,
$$

where $U_{L F}(c)$ is the laissez-faire equilibrium payoff of type $c$ and $U(c)$ is the payoff in the mechanism.

It turns out that the optimal Pareto-improving policy always shares an important feature of this example: an atom of types should trade at time 0 . Moreover, in many environments the policy described above is in fact the optimal Pareto-improving policy. We obtain the following characterization:

Theorem 2 (i) For every $r>0, F(c)$, and $v(c)$, the optimal Pareto-improving intervention has an atom of types trading at $t=0$.

(ii) If $(v(c)-c) f(c)$ is (weakly) decreasing, then an optimal Pareto-improving intervention can be implemented as a competitive equilibrium with taxes:

$$
\begin{aligned}
\tau_{0} & \leq 0 \\
\tau_{t} & \geq H \text { for } t \in(0, \Delta) \\
\tau_{t} & =0 \text { for } t \geq \Delta,
\end{aligned}
$$

so that the subsidy $\tau_{0}$ exhausts all the government's budget and $H$ is high enough so that there is no trade for $t \in(0, \Delta)$. In equilibrium, types below a threshold $c^{*}$ trade at time 0 and types above $c^{*}$ trade at the same times and prices as in the laissez-faire economy (so that $\Delta=t\left(c^{*}\right)$ in the laissez-faire).

Using the envelope theorem, we can express constraints (8) alternatively as

$$
\int_{c}^{1}(x(\tilde{c})-y(\tilde{c})) d \tilde{c}+U(1)-U_{L F}(1) \geq 0 \text { for all } c,
$$

where $y(c) \equiv e^{-r t(c)}$ is the discount factor related to the time type $c$ trades in the laissez-faire equilibrium, $t(c)$. Part (i) is immediate by noticing that trading with small $c$ immediately contribute positively to the budget constraint and the objective function, and relax the IR constraints.

For the proof of part (ii), the key step is the observation that the derivative with respect to $x(c)$ of the Lagrangian $\left(L_{P}(c)\right)$ that combines the objective function and the budget constraint is strictly decreasing under our assumption that $(v(c)-c) f(c)$ is decreasing. It is then optimal that all types for which $L_{P}(c)$ is positive trade immediately. For all the 
other types, the Lagrangian is maximized by postponing their trade as much as possible. That delay is limited however by the IR constraints (8), which require $x(c) \geq y(c)>0$ for some types even if the $L_{P}(c)$ at those types is negative. ${ }^{21}$ The regularity assumption in Theorem 2 implies that $L_{P}(c)$ is monotone. We use this monotonicity to show that it is welfare-improving to increase $x(c)$ for small types and decrease it by the same amount for large types if it allows us to still keep IR constraints satisfied. Such local variation argument pushes the IR constraints to be satisfied exactly for all types for which the $L_{P}(c)$ is negative. So the optimal allocation is $x(c)=1$ for types $c \leq c^{*}$ and $x(c)=y(c)$ for all higher types. Finally, $c^{*}$ is such that all budget is spent at the trade at time zero and the price is high enough that type $c^{*}$ is indifferent between trading at that price and waiting till $t\left(c^{*}\right)$ to trade at $v\left(c^{*}\right)$. This allocation can be implemented with the tax policy described above.

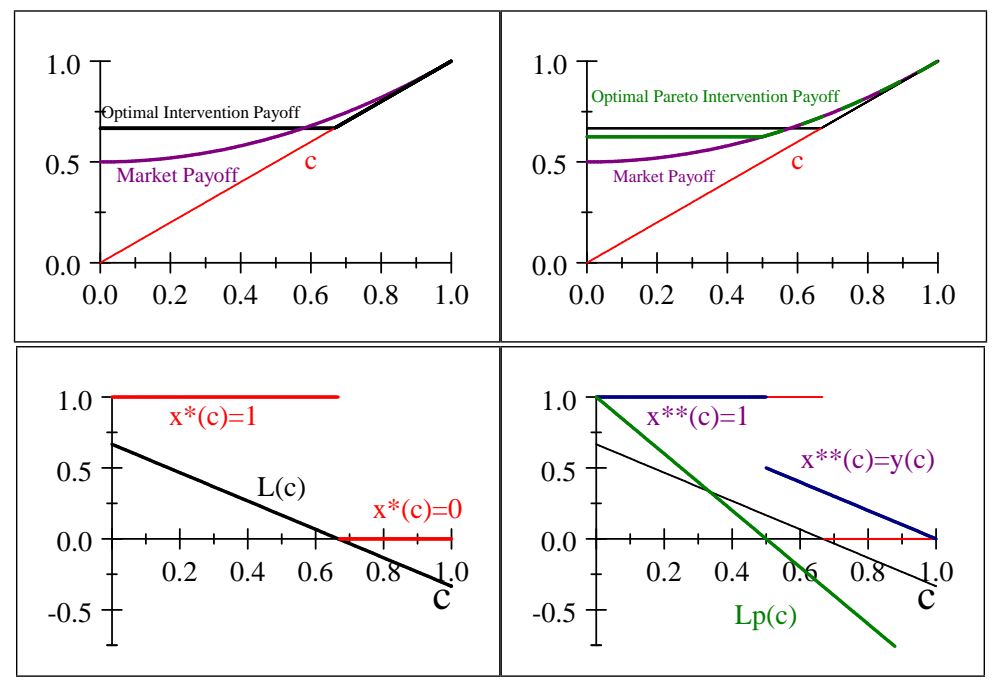

Figure 5: Payoffs and Policy with (right) and without (left) Pareto Improvement Constraint

The left panels of Figure 5 above illustrate for the case $F(c)=c$ and $v(c)=\frac{1+c}{2}$ the payoffs and optimal policy without the constraint of having to make each type at least as well off as in the laissez-faire economy. The panels on the right show how the policy and payoffs are modified to ensure the intervention is a Pareto improvement. $L_{P}(c)$ and $L(c)$ are respectively the derivatives of the Lagrangian with and without the additional constraint that the policy must lead to a Pareto improvement.

\footnotetext{
${ }^{21}$ Without these constraints, Theorem 1 established that optimal mechanism has trade only at time 0 under a weaker regularity assumption.
} 


\subsection{Jump Starting the Market}

We now show via an example that if the adverse selection problem is sufficiently severe, even the best budget-neutral intervention is incapable of generating any trade. Consider the following example: $F(c)$ is uniform in $[0,1]$ and

$$
v(c)=\left\{\begin{array}{cl}
1.5 c & \text { if } c<\frac{1}{2} \\
\frac{1+c}{2} & \text { if } c \geq \frac{1}{2}
\end{array}\right.
$$

This example satisfies the regularity condition. Hence, Theorem 1 implies that the optimal budget-neutral intervention would induce trade only at $t=0$. Unfortunately, as in Akerlof's original example, even if there is only one opportunity to trade, we get complete market breakdown. ${ }^{22}$ The equilibrium cutoff type $\kappa$ must satisfy:

$$
\kappa=p_{0},
$$

but the zero-profit condition is

$$
p_{0}=E[v(c) \mid c<\kappa] .
$$

Since in this example $E[v(c) \mid c<\kappa] \leq \frac{3}{4} \kappa$, the only solution is $\kappa=0$.

Now suppose the government allocates a budget $b \geq 0$ to bailout this market. Suppose it uses it to subsidize the initial trades with a proportional subsidy $s$. When $b<\frac{1}{16}$ the optimal intervention has $s=\frac{1}{3}$ (and prohibitively high taxes after $t=0$ ), types below $\kappa_{0}=2 \sqrt{b}$ trade at $t=0$ and buyers pay $p_{0}=\frac{3}{2} \sqrt{b}$ per unit. Total gains from trade (net of transfers) are:

$$
S(b)=\int_{0}^{2 \sqrt{b}}(0.5 c) d c=b .
$$

Since the buyers still break-even, every dollar the government spends increases the welfare of the sellers by 2 dollars, one from the direct transfer and one from the improvement in the efficiency of the market (if $b>\frac{1}{16}$, the marginal effect is even higher). Thus, if the deadweight loss associated with raising taxes from other markets is not too large, bailouts are welfare-improving.

Remark 1 It is important to note that the exact form of the intervention is not important, as long as it leads to the same discounted probability of trade and gives the same surplus to

\footnotetext{
${ }^{22}$ The classic example from Akerlof has $v(c)=1.5 c$ and no trade in (static) equilibrium. We keep close to his example, but modify $v(c)$ slightly to have $v(1)=1$. Note that it implies even less gains from trade than when $v(c)=1.5 c$.
} 
the lowest type. ${ }^{23}$ A proportional subsidy, as assumed for concreteness in the paper, or a government guarantee on the payoff of the assets, as implemented during the crisis with the Public-Private Investment Program for Legacy Assets, would have equivalent effects. The outright purchase of assets done with the TARP program is also equivalent, as long as we take into account the proceeds in the budgeting for the program, that is, consider just the expected net cost of the program as the government's budget. ${ }^{24}$

\section{Extensions and Discussion}

In this Section we discuss three important additional considerations. First, we extend our model to account for the possibility of additional sellers arriving after $t=0$. In this case the optimal policy involves controlling the price in the market rather than the times at which the market is open. Next, we point out that if the government cannot commit, it might want to use its budget constraint as a way to induce commitment to high taxes in the future by spending resources at $t=0$. Lastly, we discuss the importance of the government acting quickly since delayed interventions can be welfare reducing.

\subsection{Arrival of Additional Sellers.}

So far we have assumed that all the sellers are present at time zero. A natural concern, particularly when the optimal policy calls for allowing trade only at $t=0$, is what happens when we account for the possibility that additional sellers can arrive over time.

In order to address this concern, we augment our model and assume that additional sellers with a mass of size $m_{t}$ might arrive (stochastically or deterministically) over time. We assume that all of the sellers' types, regardless of their time of arrival, are drawn from the same type distribution $F(c)$.

Theorem 3 Suppose sellers of mass $m_{t}$ arrive to the market at a Poisson rate $\rho_{t}$. If the environment is regular, the optimal budget-balanced (on average) mechanism can be implemented with a government program in which the government offers to buy all securities in the market at a constant $p^{*}$ (and does not allow trade at higher prices in the market).

If the government does not have a budget, then it can simply decentralize the optimal policy

\footnotetext{
${ }^{23}$ This result is referred to in the mechanism design literature as "Payoff Equivalence".

${ }^{24}$ As we discussed in the Introduction, the government proceeds can either come from holding the assets to maturity or alternatively from creating a portfolio and selling its shares.
} 
by restricting the price in the market to $p^{*}$. With a budget, a decentralization involves a combination of a fixed market price and a proportional subsidy.

Proof. Consider a relaxed problem in which arrivals are observable and the government can design cohort-specific policies. That is, the government chooses for each cohort, conditional on it arriving at time $t$, policy $x_{t}(c)$ (and the corresponding $P_{t}(c)$ ) to maximize:

$$
\begin{aligned}
& \max _{x_{t}(c)} E_{\rho} \int_{0}^{\infty} 1_{t} m_{t} e^{-r t}\left(\int_{0}^{1} x_{t}(c)(v(c)-c) f(c) d c\right) d t \\
& \text { s.t. } \\
0 \leq & E_{\rho} \int_{0}^{\infty} 1_{t} m_{t} e^{-r t}\left[\int_{0}^{1}\left(x_{t}(c)(v(c)-c)\right) f(c) d c-\int_{0}^{1} x_{t}(c) F(c) d c\right] d t+b,
\end{aligned}
$$

where $1_{t}$ is the indicator function for the arrival at time $t$ and the expectations are over the arrival times of the new sellers. The Lagrangian of this problem is:

$$
\max _{x_{t}(c)} E_{\rho} \int_{0}^{\infty} 1_{t} m_{t} e^{-r t}\left(\int_{0}^{1} x_{t}(c)[(v(c)-c) f(c)(1+\Lambda)-\Lambda F(c)] d c\right) d t+\Lambda b .
$$

It is immediate that the optimal allocation $x_{t}(c)$ is the same for all times of arrivals. Since the environment is regular, by Theorem 1 it has the bang-bang property: $x_{t}(c)=1$ for $c$ below a threshold $c^{*}$ and $x_{t}(c)=0$ above $c^{*}$ (and the threshold is constant over time). A way to implement this constant trading threshold is to set a constant price $p^{*}=c^{*}$. Under this policy, arriving sellers would either sell immediately or not at all. Hence this policy remains optimal even if the government cannot condition on the arrival times.

The proof can be trivially extended to an uncertain mass of arriving sellers (i.e., $m_{t}$ being a random variable) or to deterministic arrivals. The optimal price is set in a way to exhaust the budget on average. It is important for the policy that the government controls the private market, not allowing trades at prices higher than $p^{*}$. Otherwise, if the arrival time of a seller was observed by the market, the high types would be able to get a better price after some delay, undermining the optimal mechanism. In other words, even though the optimal mechanism now has trade potentially in every period, it still is optimal to restrict trade in the private markets.

We finish by pointing out that Theorem 3 is related to Sobel (1991) who studies a monopoly problem under commitment with the arrival over time of new consumers from a common constant distribution. He shows that the optimal monopoly price is constant over time. Our result is similar, but it requires additional assumptions about the distribution of types and gains from trade, namely the regularity condition. This assumption is needed because 
our problem has interdependent values between buyers and sellers, and the government is required to balance the inter-temporal budget. In other settings, these distinctions can lead to very different outcomes. ${ }^{25}$

\subsection{Commitment via the Budget}

An important assumption in our model is that the government can commit to future taxes. Without commitment, the government would like to revise its tax policy after the initial round of trade, to allow even more trade. Full analysis of the case without commitment is left for future work, but we illustrate with an example how the budget constraint can serve as a commitment device.

Recall our example from Section 3 with $F(c)=c$ and $v(c)=\frac{1+c}{2}$. It satisfies our regularity condition and hence the optimal policy would have trade only at time $t=0$. Assuming the government has no resources $(b=0)$, the optimal policy would entail $p=2 / 3$ and $c^{*}=2 / 3$. The government would be tempted to lower taxes and let trade take place again after time zero. If the sellers anticipated these future opportunities to trade, they would alter their behavior at $t=0$, reducing the efficiency of trade. While it may not be possible to implement the optimal policy without commitment to future taxes, if the government has commitment to satisfy its budget, it may be possible to improve upon laissez-faire. In particular, the government could pick taxes $\tau_{t}^{*}$ that would maximize the government's tax proceeds in the future, borrow against them, and spend all the money subsidizing trades at $t=0$. The government would thus need to indeed keep the taxes at $\tau_{t}^{*}$ in order to be able to repay its debts. In this way the government still induces a lot of trade to take place at $t=0$ and, in our example, can obtain about half of the welfare gains they could have obtained with full commitment.

\subsection{The Cost of Delaying Interventions}

In practice it might take time for the government to act upon a crisis. In this section we show that speed is often of the essence. Not only is it usually optimal to act immediately, as established by Theorem 1, but delayed interventions can actually decrease surplus compared to the laissez-faire equilibrium. In particular, we show that if the government is expected to provide a bailout at some future time, it slows down (or even shuts down) trade prior to the intervention. Even though the bailout has a positive direct effect on efficiency, it also creates

\footnotetext{
${ }^{25}$ For example, in a bargaining problem (without commitment), with independent values we get immediate trade (the Coase conjecture result) while with interdependent values the equilibrium typically exhibits delay.
} 
this negative endogenous/equilibrium effect of delay due to anticipation. The net effect can be negative: the equilibrium welfare with a delayed subsidy can be lower than absent any intervention.

We show this claim via two examples using the benchmark example from Section 3, with $v(c)=\frac{1+c}{2}$ and $F(c)=c$. We consider first the case of a deterministic date for the intervention and then the case when the timing of the intervention is uncertain.

\subsubsection{Delayed Interventions at Announced Date}

Consider the following policy:

$$
\tau_{t}^{T}=\left\{\begin{array}{c}
-s \text { for } t=T \\
0 \text { otherwise }
\end{array}\right.
$$

The government has a dynamic budget constraint $e^{-r T} s p_{T}\left|K_{T}\right| \leq b$, where $\left|K_{T}\right|$ is the measure of types that trade at $T$ and $p_{T}$ is the market price buyers pay at $T$, so that the left hand side is the time-zero present value of the total subsidy at $T$.

For any size of the intervention, $b>0$, there exists a $\bar{T}$ such that if $T \leq \bar{T}$ there is no trade in equilibrium until $T$ since all sellers prefer to wait for the subsidy than to trade immediately. Thus, in this range of $T$ the competitive equilibrium has atom of trade at $T$ followed by smooth trading as in the laissez-faire equilibrium. The following conditions pin down the equilibrium for $T \leq \bar{T} \cdot{ }^{26}$

First, denote by $\kappa$ the highest type trading at $T$. This type has to be indifferent between trading at the subsidized price and trading after the subsidy is removed:

$$
p_{T}(1+s)=p_{T^{+}}=v(\kappa)
$$

where the second equality follows because after $T$ the equilibrium coincides with the laissezfaire equilibrium with a boundary condition $k_{T}=\kappa$ (and hence the zero-profit condition with smooth trading implies $\left.p_{t}=v\left(k_{t}\right)\right)$. The zero-profit condition for prices at $T$ is:

$$
p_{T}=E[v(c) \mid c \leq \kappa]
$$

\footnotetext{
${ }^{26}$ When $T$ is large, the analysis is more complex. The equilibrium then has continuous trading until some time $T^{*}$; from $T^{*}$ to $T$, the market shuts down waiting for the subsidy, an atom of sellers trade at $T$, and smooth trading follows from then on.
} 
Finally, the budget constraint is:

$$
e^{-r T} p_{T} s \kappa=b
$$

From these equations (using $v(c)$ and $F(c)$ from the benchmark example) we obtain:

$$
\kappa=2 \sqrt{b e^{T r}} .
$$

Assuming that $b$ is small enough that $\kappa<1$, after $T$ trade is smooth and we can use the characterization of the laissez-faire equilibrium in Section 3 to compute:

$$
k_{t}=1-(1-\kappa) e^{-r(t-T)} .
$$

Inverting it yields the time at which types $k>\kappa$ trade:

$$
\tilde{t}(k)=-\frac{1}{r}\left(\ln \frac{1-k}{1-\kappa}\right)+T .
$$

That completes the characterization of the equilibrium. ${ }^{27}$

Present value of the gains from trade in equilibrium is:

$$
S(b, T<\bar{T})=e^{-r T}\left(\int_{0}^{\kappa}\left(\frac{1-c}{2}\right) d c+\int_{\kappa}^{1}\left(\frac{1-c}{1-\kappa}\right)\left(\frac{1-c}{2}\right) d c\right) .
$$

Since $b=0$ corresponds to no intervention, we have $S(0, T)=S_{L F}=\frac{1}{6}$.

How does delay, $T$, affect gains from trade? ${ }^{28}$ There are two opposing effects. On the one hand, later subsidy implies that there is more delay until the market starts trading at $T$. On the other hand, since the unused budget earns interest, it allows for more efficient trade at $T$ and afterwards. It turns out that in our benchmark case the first force is stronger. In particular, for $b>0$ and $T \leq \bar{T}$ and $2 \sqrt{b e^{T r}}<1$ we get the following result:

Proposition 2 Assume $v(c)=\frac{1+c}{2}$ and $F(c)=c$.

1) Despite the government being able to save at rate $r$, the equilibrium welfare is decreasing in $T, \frac{\partial S(b, T)}{\partial T}<0$.

\footnotetext{
${ }^{27}$ It implies $\bar{T}$ is the solution to $e^{-r \bar{T}}(1+s) p_{T}=v(0)$ which simplifies to a solution of $b=\frac{1}{4} \frac{(1-z)^{2}}{z}$ where $z=e^{-r \bar{T}}$

${ }^{28}$ The effect of $b$ is obvious: if $b$ is small enough so that not all types trade at $T, S(b, T)$ is increasing in $b$ for all $T$, because higher $b$ uniformly speeds up trade.
} 
2) Moreover, there exists $T^{*}<\bar{T}$ such that the subsidy delayed by more than $T^{*}$ destroys surplus, that is $S(b, T)<S_{L F}$ for $T \in\left(T^{*}, \bar{T}\right)$.

In words, delay is costly despite the budget growing with delay. Even more surprisingly, the second part of the proposition states that the decrease in the surplus can be so large as to drive the total surplus below the surplus with no intervention. For example with $b=\frac{1}{10}$ and $r=1$, if $T>T^{*}=0.387$ then $S(b, T)<\frac{1}{6}=S_{L F}$ (in this case $\bar{T}=0.622$ ). This is illustrated in Figure 5.

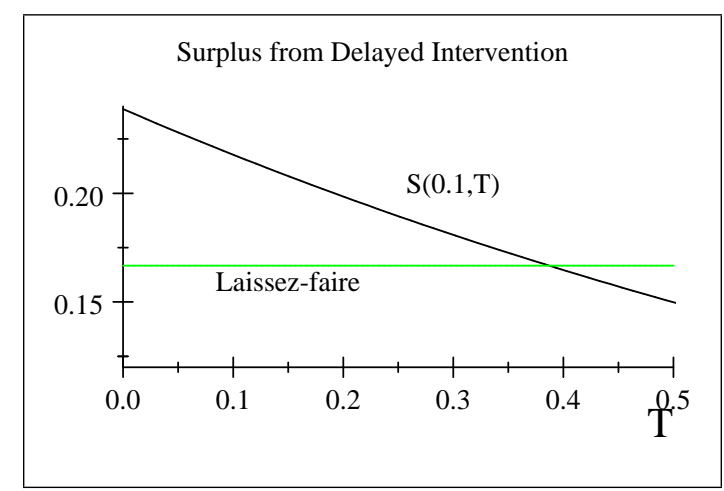

Figure 5: The Cost of Delayed Interventions.

\subsubsection{Delayed Interventions with Uncertain Timing}

In practice the market might expect the possibility of a government subsidy but be uncertain about its timing. We argue that this creates incentives to wait for the arrival of the intervention and may be detrimental to welfare even taking into account the benefits of the subsidy if it materializes.

To illustrate this problem, we analyze a model in which the government intervention arrives at a random time as a Poisson process with intensity $\lambda$. Suppose that when it finally intervenes, the government subsidizes trade sufficiently that all types trade (by offering sufficient subsidy for $\left.p_{t}(1+s)=1\right)$.

The equilibrium dynamics depend crucially on the level of $\lambda$. If $\lambda$ is small, there will be trade even before the government subsidy arrives. If it is high, the market will shut down completely until the arrival of the subsidy.

We start with the first, more interesting case. If $\lambda<r$, then trade is smooth until arrival of the subsidy. Equilibrium cutoffs $k_{t}$ are characterized by the following differential equation 
(with a boundary condition $k_{0}=0$ ):

$$
r\left(v\left(k_{t}\right)-k_{t}\right)=v^{\prime}\left(k_{t}\right) \dot{k}_{t}+\lambda\left(1-v\left(k_{t}\right)\right) .
$$

The left-hand side is the familiar discounting cost of not taking the price today. The first term on the right-hand side is the familiar increase in price in case the intervention does not arrive (as before, both use the zero-profit condition $p_{t}=v\left(k_{t}\right)$ ). The new term is the last term on the right-hand side: by delaying trade, the current cutoff type can hope to receive the subsidized price 1 instead of the non-subsidized price $v\left(k_{t}\right)$.

A higher $\lambda$ has two opposing welfare effects. The direct effect is that it speeds up the arrival of the subsidy, which increases welfare. The indirect, equilibrium effect, is that it slows down trade before arrival and it decreases welfare (the indirect effect can be seen from (9) since $\dot{k}_{t}$ that solves it is decreasing in $\lambda$ ).

Connecting this observation to real-life events, market participants' beliefs that Federal Reserve and/or the US Treasury would intervene in some financial markets, might have contributed to the reduction of trade volume in some of the those markets after the collapse of Lehman Brothers. While asymmetric information was likely the primary culprit, the expectations of future actions could make it worse.

Using $v(c)$ and $F(c)$ from the benchmark example, the differential equation (9) simplifies to:

$$
(r-\lambda)\left(1-k_{t}\right)=\dot{k}_{t} .
$$

After solving it and computing total welfare, we can show that the negative, indirect effect always dominates (unless $\lambda$ is so high that the market shuts down completely):

Proposition 3 Assume $v(c)=\frac{1+c}{2}$ and $F(c)=c$. Suppose the government subsidy that induces first-best trade arrives at a Poisson rate $\lambda$.

1) If $\lambda<r$, there is trade in equilibrium even before the subsidy arrives and the equilibrium welfare is strictly decreasing in $\lambda$.

2) If $\lambda>r$ then the market shuts down in the anticipation of the subsidy and equilibrium welfare is increasing in $\lambda$ (as $\lambda \rightarrow \infty$ the surplus converges to first-best). In that range, the surplus is higher than the laissez-faire equilibrium surplus if and only if $\lambda$ is sufficiently higher than $r$.

In words, Proposition 3 demonstrates that, over a large range of arrival rates, the anticipation of future intervention reduces welfare in our benchmark example. In fact, for the 
delayed subsidy to have a hope at improving welfare, the arrival rate has to be sufficiently high so that the market closes down completely.

Figure 6 illustrates the results for $r=1$.

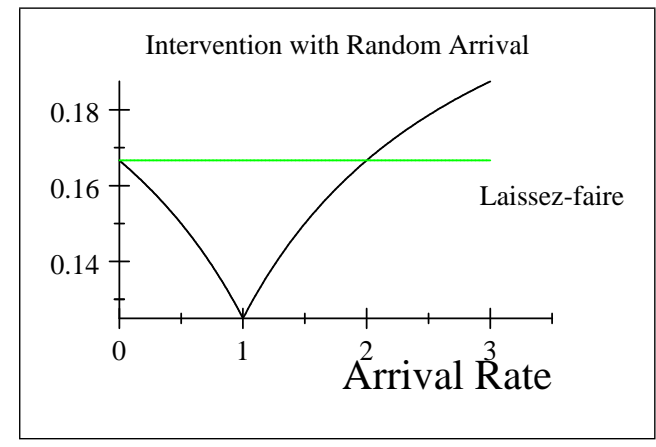

Figure 6: Cost of Random Arrival of Subsidy.

The intuition behind the second part of the above proposition is straightforward. For $\lambda$ sufficiently large there will not be any trade until the government intervenes. Hence, there is only the first, direct effect. Since equilibrium surplus is continuous in $\lambda$ and it decreases from $S_{L F}$ as $\lambda$ increases from 0 to $r$, it has to increase sufficiently more to recover back to $S_{L F}$.

\section{Conclusions}

In this paper we have analyzed government interventions in a dynamic market with asymmetric information. Our main finding is that even without the use of subsidies, efficiency can be improved over the laissez-faire equilibrium. Setting high taxes for a short period, after an initial tax-exempt auction or trading window, induces more sellers to trade early. This bunching improves the average quality traded in the market which in turn leads to higher prices. As prices increase, more sellers choose to trade early, further improving welfare.

Remarkably, under a fairly commonly used regularity condition, the optimal government policy is to set high taxes for $t>0$, effectively shutting down private markets. Of course these results have to be interpreted with caution. As we show there might be natural reasons to depart from this recommendation. First, this recommendation leads to both winners and losers and can therefore lack the support necessary to be implemented. Instead, a short closure can actually be Pareto improving. That is, all seller types are made better off. We 
also show that if more sellers arrive at later dates, it is no longer optimal to have high taxes for $t>0$. Instead, price controls should be implemented. By keeping the price constant, the sellers, regardless of when they arrive, effectively see only one opportunity for trade. This leads to the same bunching of types within a cohort that is achieved via high taxes, but with the necessary flexibility to accommodate arrivals of future cohorts.

Freeing the government from the requirement that its intervention must be budget-neutral, i.e. allowing for bailouts, can be very valuable. Moreover, although the particular form in which the subsidy is provided is not important, its timing is. Subsidies can greatly enhance welfare when provided immediately or quickly after the shock, but they can even destroy surplus if they are implemented with a delay and anticipated by market participants.

\section{Appendix}

\section{Equilibrium computation for Initial Subsidy followed by a Constant Permanent} Tax:

The equilibrium conditions are as follows. First, cutoff type at time $0, \kappa \equiv k_{0^{+}}$, must be indifferent between pooling with lower types and getting the initial subsidized price $p_{0}$, or waiting an instant to separate from them and getting a higher price but being taxed:

$$
p_{0}(1+s)=\max \{\underbrace{v(\kappa)}_{=p_{0^{+}}}(1-\tau), \kappa\} .
$$

where the maximization captures the two possibilities: there will either be trade after $t=0$ (with buyers paying $p_{0^{+}}=v(\kappa)$ ) or no trade at all.

Second, the buyer zero profit condition at time $t=0$ is:

$$
p_{0}=E[v(c) \mid c \in[0, \kappa]] .
$$

The unique solution to (10) and (11) pins down the equilibrium $\kappa$ and price $p_{0}$ given $(s, \tau)$.

Third, if there is any more trade after time $t=0$ (i.e. if $\tau$ is not too high) it must be smooth. The same reasoning as in the laissez-faire equilibrium holds after time zero. Equilibrium is then pinned down by the sellers' indifference condition:

$$
r\left(p_{t}(1-\tau)-k_{t}\right)=(1-\tau) \dot{p}_{t}
$$


and the zero-profit condition $p_{t}=v\left(k_{t}\right)$. Using the assumed form of $v(c)$ and given a boundary condition $\kappa$, the unique solution of this differential equation is:

$$
k_{t}=\frac{1-\tau}{1+\tau}-\left(\frac{1-\tau}{1+\tau}-\kappa\right) e^{-r \frac{1+\tau}{1-\tau} t}
$$

Inverting it, we get the following expression for the time at which each cutoff type $k$ trades:

$$
\tilde{t}(k)=\frac{1}{r} \frac{\tau-1}{\tau+1}\left(\ln \frac{k(1+\tau)+\tau-1}{\kappa(1+\tau)+\tau-1}\right) \text { for } k \in\left[\kappa, \frac{1-\tau}{1+\tau}\right] .
$$

Note that because of the tax, types such that $(1-\tau) v(c) \leq c$ do not trade in equilibrium. That completes the characterization of the equilibrium for any $(s, \tau)$.

Finally, to verify that in equilibrium the intervention is budget-neutral, we require that for any fixed $\tau$ the subsidy $s$ satisfies:

$$
s p_{0} \kappa=\tau \int_{\kappa}^{1} e^{-r \tilde{t}(k)} \frac{1+k}{2} d k .
$$

The unique positive solution $\left(s, \kappa, p_{0}\right)$ to $(10),(11),(12)$ pins down the unique competitive equilibrium in this example. With it we can calculate the total surplus associated with a given tax rate $\tau$ :

$$
S(\tau)=\int_{0}^{\kappa}(v(c)-c) d c+\int_{\kappa}^{1} e^{-r \tilde{t}(k)}\left(v\left(k_{t}\right)-k_{t}\right) d k
$$

\section{Omitted Proofs:}

Proof of Proposition 1. First, note that our requirement $p_{t} \geq v\left(k_{t}\right)$ implies that there cannot be any atoms of trade, i.e., that $k_{t}$ has to be continuous. Suppose not, so that at time $s$ types $\left[k_{s}, k_{s^{+}}\right]$trade and $k_{s}<k_{s^{+}}$. Then at time $s+\varepsilon$ the price would be at least $v\left(k_{s^{+}}\right)$ while at $s$ the price would be strictly smaller to satisfy the zero-profit condition (1E). If so, then for small $\varepsilon$ all types in $\left[k_{s}, k_{s^{+}}\right]$would be better off not trading at $s$, a contradiction. Therefore we are left with processes such that $k_{t}$ is continuous and $p_{t}=v\left(k_{t}\right)$ at any time such that $\dot{k}_{t^{+}} \geq 0$. If $k_{t}$ is strictly increasing over time, we need that $r\left(p_{t}-k_{t}\right)=\dot{p}_{t}$ : if price was rising faster, current cutoffs would like to wait, a contradiction. If prices were rising slower, over any time interval starting at $s$, there would be an atom of types trading at $s$, another contradiction. So the only remaining possibility is that $k_{t}$ is constant over some 
interval $\left[s_{1}, s_{2}\right]$. Since the price at $s_{1}$ is $v\left(k_{s_{1}}\right)$ and the price at $s_{2}$ is $v\left(k_{s_{2}}\right)$, if there is indeed no trade in that time interval, then $p_{s_{1}}=p_{s_{2}}$. But then there exists a positive measure of types $k>k_{s_{1}}$ such that

$$
v\left(k_{s_{1}}\right)>\left(1-e^{r\left(s_{2}-s_{1}\right)}\right) k+e^{r\left(s_{2}-s_{1}\right)} v\left(k_{s_{1}}\right) .
$$

Since after $s_{2}$ there are no atoms of trade, the equilibrium continuation payoff of types $k>k_{s_{1}}$ is smaller than $\left(1-e^{r\left(s_{2}-s_{1}\right)}\right) k+e^{r\left(s_{2}-s_{1}\right)} v(k)$, since these types trade at price $v(k)$, but later than $t=s_{2}$. Since $v(k)$ is continuous, there exists an $\varepsilon$ such that types $k \in\left[k_{s_{1}}, k_{s_{1}}+\varepsilon\right]$ would strictly prefer to trade at $t=s_{1}$ than to follow the postulated equilibrium. That leads to the final contradiction.

Proof of Theorem 1 and Lemma 1. We use mechanism design approach to establish the two results. The mechanism designer chooses a direct revelation mechanism that maps reports of the sellers to (i) a probability distribution over times they trade, to (ii) transfers from the buyers to the mechanism designer, and to (iii) transfers from the designer to the sellers. The constraints on the mechanism are: incentive compatibility for the sellers (to report truthfully); individual rationality for the sellers and buyers (sellers prefer to participate in the mechanism rather than hold the asset forever and the buyers do not lose money on average); and that the mechanism designer does not lose more money on average than its budget, $b$. We then characterize a tax policy and a corresponding equilibrium that implements the same outcome as the optimal mechanism we find.

Let $G_{t}(c)$ denote, for a given type, the distribution function over the times of trade. Define:

$$
x(c)=\int_{0}^{T} e^{-r t} d G_{t}(c) .
$$

Since all players are risk neutral, the surplus and all constraints of the mechanism depend on $G_{t}(c)$ only via $x(c)$. Hence, we can focus on general direct revelation mechanisms described by two functions: $x(c)$ and $P(c)$, where $P(c)$ is the expected net present value of the transfer received by the seller reporting type $c$.

The objective function of the mechanism designer is to maximize total surplus

$$
\max _{x(c) \in[0,1], P(c)} \int x(c)(v(c)-c) f(c) d c .
$$

We now describe the constraints. 
The mechanism yields the following sellers' value function:

$$
\begin{aligned}
U(c) & =P(c)+(1-x(c)) c \\
& =\max _{c^{\prime}} P\left(c^{\prime}\right)+\left(1-x\left(c^{\prime}\right)\right) c .
\end{aligned}
$$

Using the envelope condition we can express it as:

$$
U(c)=U(1)-\int_{c}^{1}(1-x(c)) d c
$$

Seller IR constraint is $U(c) \geq c$; in the optimal mechanism it binds at $c=1 .{ }^{29}$ Incentive compatibility for the sellers is satisfied if and only if the envelope formula (16) holds and $x(c)$ is weakly decreasing.

The buyers are willing to pay on average at most $\int_{0}^{1} x(c) v(c) f(c) d c$. An optimal mechanism leaves the buyers with no surplus (since we could reduce the payment to the buyers and use the savings to increase efficiency of trade). Therefore, the budget constraint of the designer is:

$$
\int_{0}^{1}(x(c) v(c)-P(c)) f(c) d c+b \geq 0 .
$$

From (14), we can write $P(c)$ as:

$$
P(c)=U(c)-(1-x(c)) c
$$

Substituting this for $P(c)$ in the budget constraint, we can express it as a function of the allocation alone:

$$
\int_{0}^{1}(x(c)(v(c)-c)) f(c) d c-\int_{0}^{1}(U(c)-c) f(c) d c+b \geq 0
$$

Next, we can use $(16), U(1)=1$, and integration by parts to write the second term as:

$$
\int_{0}^{1}(U(c)-c) f(c) d c=\int_{0}^{1} x(c) F(c) d c .
$$

\footnotetext{
${ }^{29} \mathrm{By}(16)$, if the IR constraint is satisfied at $c=1$, it is satisfied for all types. IR binds at $c=1$ since otherwise the mechanism designer could reduce $P(c)$ by a constant and still satisfy all constraints.
} 
This yields the final form of the budget constraint:

$$
\int_{0}^{1} x(c) v(c) f(c) d c-\int_{0}^{1} x(c)\left(c+\frac{F(c)}{f(c)}\right) f(c) d c+b \geq 0 .
$$

The first term of the constraint is the revenue the designer can obtain from the buyers and the second term is the amount he has to pay the sellers to participate in the mechanism that includes their information rent.

We now optimize (13) subject to (17). The derivative of the Lagrangian with respect to $x(c)$ is:

$$
L(c)=(v(c)-c) f(c)(1+\Lambda)-\Lambda F(c),
$$

where $\Lambda>0$ is the Lagrange multiplier on (17).$^{30}$

Note that $L(c)$ is (weakly) positive for $c=0$ (strictly if $v(0)>0$ ) and negative for $c=1$ (assuming that $b$ is not too large, so that the budget constraint is binding, and hence $\Lambda>0$ ).

If $\frac{f(c)}{F(c)}(v(c)-c)$ is decreasing (which is our regularity assumption), then $L(c)$ crosses zero only once for any $\Lambda>0$. Let $c^{*}$ be the largest solution to

$$
L(c)=0 .
$$

An optimal $x(c)$ is then:

$$
x^{*}(c)=\left\{\begin{array}{l}
1 \text { if } c \leq c^{*} \\
0 \text { if } c>c^{*}
\end{array}\right.
$$

Since $x^{*}(c)$ is monotone, a mechanism with this allocation (and appropriate $P(c)$ ) is incentive compatible. The largest $c^{*}$ that satisfies the budget constraint (17) is the largest solution of:

$$
E\left[v(c) \mid c \leq c^{*}\right]+\frac{b}{F\left(c^{*}\right)}=c^{*} .
$$

To see this, note that the LHS is the average price per asset the buyers are willing to pay for assets below $c^{*}$, plus the total budget per unit of trade (total volume of trade is $F\left(c^{*}\right)$ ). The RHS is the reservation price of the $c^{*}$ seller.

A tax policy $\tau_{0}=1-\frac{c^{*}}{E\left[v(c) \mid c \leq c^{*}\right]}=-\frac{b}{\int_{0}^{c^{*}} v(c) f(c) d c}$ (and $\tau_{t}=H$ for $t>0$ ) induces an equilibrium such that there is trade only at time zero and that equilibrium satisfies (18) for some $c^{*}$. To finish the proof, we need to show that the solution to (18) exists and if the environment is strictly regular, the solution is unique.

\footnotetext{
${ }^{30} \Lambda$ is strictly positive in the solution for sufficiently small $b$. Otherwise the budget constraint would not be binding and we would get $x(c)=1$ for all $c$ (first-best), violating (17).
} 
1) Existence. To see that there exists at least one solution to (18) note that

$$
E[v(c) \mid c \leq k]-k+\frac{b}{F(k)}
$$

is continuous in $k$, positive at $k$ close to zero, and negative at $k=1$ (as before, assuming $b$ is not sufficient to achieve full efficiency). So there exists at least one solution.

2) Uniqueness. To see that the solution is unique under the regularity assumption, note that the derivative of (19) at any $k$ is

$$
\frac{f(k)}{F(k)}\left(v(k)-E[v(c) \mid c \leq k]-\frac{b}{F(k)}\right)-1 .
$$

When we evaluate it at points where (18) holds, the derivative is

$$
\frac{f(k)}{F(k)}(v(k)-k)-1
$$

and that is by assumption decreasing in $k$.

Suppose that there are at least two solutions and select two: the lowest $k_{L}$ and secondlowest $k_{H}$. Since $k_{L}$ is the lowest solution, at that point the curve (19) must have a weakly negative slope (since the curve crosses zero from above). However, our assumption implies that curve has even strictly more negative slope at $k_{H}$. That leads to a contradiction since by assumption between $\left[k_{L}, k_{H}\right]$ expression (19) is negative, so with this ranking of derivatives, it cannot become 0 at $k_{H}$. That completes the proof of Theorem 1 .

Regarding Lemma 1, consider $L(c)$ that crosses 0 more than once. Suppose that there exists an optimal $x(c)$ that takes more than three values, $\{0, \chi, 1\}$. The argument in the text explains why, if $x(c)$ is a step function, we can achieve at least as high efficiency by a step function with at most two steps. The only remaining possibility is that $x(c)$ decreases continuously over some range $\left(c_{1}, c_{2}\right)$. In that case, for $x(c)$ to be optimal, $L(c)$ has to be 0 for all $c \in\left(c_{1}, c_{2}\right)$. Suppose not. Suppose it is strictly positive at $\hat{c} \in\left(c_{1}, c_{2}\right)$. Then, since $L(c)$ is continuous, it is strictly positive in a neighborhood of $\hat{c}$, say from $(\hat{c}-\varepsilon, \hat{c}+\varepsilon)$, for some $\varepsilon>0$. By increasing $x(c)$ in this range to the level $x(\hat{c}-\varepsilon)$, we maintain monotonicity of $x$ and strictly increase the Lagrangian. Analogously, if $L(c)$ is strictly negative in $(\hat{c}-\varepsilon, \hat{c}+\varepsilon)$, we can reduce $x(c)$ in this range to $x(\hat{c}+\varepsilon)$ and also get a strict improvement. So the only possibility is that $L(c)=0$ in that range. But then there exists $\hat{c} \in\left(c_{1}, c_{2}\right)$ such that if we modify $x(c)$ to be constant on $\left(c_{1}, c_{2}\right)$ at $x(\hat{c})$, the budget constraint is still binding, the 
Lagrangian is not changed, and the resulting $x(c)$ is still monotone. That means that we can always weakly improve by modifying $x(c)$ to be a step function. The reasoning in the text completes the proof that a (weakly) optimal mechanism has $x(c)$ that is a step function with at most two steps.

Finally, as we discussed in the text, this allocation can be implemented using a tax policy described there.

That the trades at time 0 have to cross-subsidize trades in time $\Delta$ follows from the observation that the second trade contributes negatively to the budget constraint and the first trade contributes positively: otherwise we would increase $x(c)$ for the second trade without violating the budget constraint.

Proof of Lemma 2. To establish that the market with $\tau^{\Delta}$ is more efficient than under laissez-faire, we show an even stronger result: that for small $\Delta$ there is more trade at $t=0$ with $\tau^{\Delta}$ than with $\tau_{t}=0$ over the time interval $[0, \Delta]$. Since under $\tau^{\Delta}$ the equilibrium after $\Delta$ is characterized by the same differential equation as under laissez-faire, if the boundary condition at $\Delta$ is higher, all types trade faster in equilibrium under $\tau^{\Delta}$.

Let $\kappa_{\Delta}$ be the highest type that trades at $t=0$ under $\tau^{\Delta}$ (i.e. $\kappa_{\Delta}=k_{0^{+}}$). Let $k_{\Delta}^{L F}$ the equilibrium cutoff at time $\Delta$ under laissez-faire. Since $\lim _{\Delta \rightarrow 0} \kappa_{\Delta}=\lim _{\Delta \rightarrow 0} k_{\Delta}^{L F}=0$ (for $\kappa_{\Delta}$ see discussion in Step 1 below), to establish that $\kappa_{\Delta}>k_{\Delta}^{L F}$ for small $\Delta$, it is sufficient to show:

$$
\lim _{\Delta \rightarrow 0} \frac{\partial \kappa_{\Delta}}{\partial \Delta}>\lim _{\Delta \rightarrow 0} \frac{\partial k_{\Delta}^{L F}}{\partial \Delta}
$$

Step 1: Characterizing $\lim _{\Delta \rightarrow 0} \frac{\partial \kappa_{\Delta}}{\partial \Delta}$.

Consider policy $\tau^{\Delta}$.

First notice that since $\tau_{t}=\tau>0$ for $t \in(0, \Delta]$ and $\tau_{t}=0$ for $t>\Delta$, for small $\Delta$ there cannot be any trade in $t \in(0, \Delta]$. Suppose not. Let $k^{*}$ be the supremum over types that trade in that time interval. All types that trade in that interval get a payoff no higher than $(1-\tau) v\left(k^{*}\right)$ (buyers would lose money if they paid more than $p=v\left(k^{*}\right)$, the government takes $\tau$ of that price, and the best case scenario is that they trade with no delay). For small $\Delta$, that payoff is smaller than $\left(1-e^{-r \Delta}\right) k^{*}+e^{-r \Delta} v\left(k^{*}\right)$. Since $p_{\Delta^{+}} \geq v\left(k^{*}\right)$, all types that trade in $(0, \Delta]$ would be strictly better off waiting for the tax to be removed, a contradiction.

When the taxes are removed after $t=\Delta$, the continuation equilibrium is unique and 
is characterized in Proposition 1 albeit with a different starting lowest type. Namely, for $t>\Delta$ :

$$
\begin{aligned}
p_{t} & =v\left(k_{t}\right) \\
r\left(v\left(k_{t}\right)-k_{t}\right) & =v^{\prime}\left(k_{t}\right) \dot{k}_{t}
\end{aligned}
$$

with a boundary condition:

$$
k_{\Delta}=\kappa_{\Delta} .
$$

The break even condition for buyers (1E) at $t=0$ implies:

$$
p_{0}=E\left[v(c) \mid c \in\left[0, \kappa_{\Delta}\right]\right] .
$$

Seller optimality (2E) implies that type $\kappa_{\Delta}$ must be indifferent between trading at this price at $t=0$ and selling for $p_{\Delta^{+}}=v\left(\kappa_{\Delta}\right)$ at $t=\Delta$ :

$$
v\left(\kappa_{\Delta}\right)-p_{0}=\left(1-e^{-r \Delta}\right)\left(v\left(\kappa_{\Delta}\right)-\kappa_{\Delta}\right) .
$$

Combining these two conditions we get that $\kappa_{\Delta}$ is a solution to:

$$
v\left(\kappa_{\Delta}\right)-E\left[v(c) \mid c \in\left[0, \kappa_{\Delta}\right]\right]=\left(1-e^{-r \Delta}\right)\left(v\left(\kappa_{\Delta}\right)-\kappa_{\Delta}\right) .
$$

For small $\Delta$ this equation has a unique solution. Using implicit function theorem we can show that:

$$
\lim _{\Delta \rightarrow 0} \frac{\partial \kappa_{\Delta}}{\partial \Delta}=\frac{2 r v(0)}{v^{\prime}(0)}
$$

Intuitively, for small $\Delta, E\left[v(c) \mid c \leq c \in\left[0, \kappa_{\Delta}\right]\right] \approx \frac{v(0)+v\left(\kappa_{\Delta}\right)}{2}$ (because we have assumed that $f(c)$ and $v(c)$ are positive and continuous). So the benefit of waiting, the left-hand side of (20), is approximately $\frac{v\left(\kappa_{\Delta}\right)-v(0)}{2}$; while the cost of waiting, the right-hand side of $(20)$, is approximately $r \Delta v(0)$. So for small $\Delta, \kappa_{\Delta}$ solves approximately

$$
\frac{v\left(\kappa_{\Delta}\right)-v(0)}{2}=r \Delta v(0)
$$

which yields $\frac{\partial \kappa_{\Delta}}{\partial \Delta}=\frac{2 r v(0)}{v^{\prime}(0)}$ as $\Delta \rightarrow 0$. 
Step 2: Characterizing $\lim _{\Delta \rightarrow 0} \frac{\partial k_{\Delta}^{L F}}{\partial \Delta}$.

Consider the laissez-faire economy. Since $k_{t}$ is defined by the differential equation

$$
r\left(v\left(k_{t}\right)-k_{t}\right)=v^{\prime}\left(k_{t}\right) \dot{k}_{t},
$$

for small $\Delta$ :

$$
k_{\Delta}^{L F} \approx r \Delta \frac{v(0)}{v^{\prime}(0)}
$$

and more precisely:

$$
\lim _{\Delta \rightarrow 0} \frac{\partial k_{\Delta}^{L F}}{\partial \Delta}=\frac{r v(0)}{v^{\prime}(0)}
$$

Summing up steps 1 and 2, we have:

$$
\lim _{\Delta \rightarrow 0} \frac{\partial \kappa_{\Delta}}{\partial \Delta}=2 \lim _{\Delta \rightarrow 0} \frac{\partial k_{\Delta}^{L F}}{\partial \Delta}
$$

which implies the claim.

\section{Step 3: Pareto Improvement}

Take any $\Delta$ such that there is no trade in $t \in(0, \Delta]$ under $\tau^{\Delta}$ and that $k_{\Delta}^{L F}<\kappa_{\Delta}$. Since all types $c>\kappa_{\Delta}$ trade at the same price but sooner in the market with $\tau^{\Delta}$ than in the laissez-faire economy, it is immediate that they all prefer the former.

Type $c=\kappa_{\Delta}$ also strictly prefers $\tau^{\Delta}$ : while he is trading at $t=0$, he has the option to trade at $p=v\left(\kappa_{\Delta}\right)$ at $t=\Delta$ while in the laissez-faire economy he trades at the same price but later. By revealed preference he is strictly better off under $\tau^{\Delta}$.

Finally consider any type $c<\kappa_{\Delta}$. All these types get the same payoff under $\tau^{\Delta}$ :

$$
U_{\tau}(c)=U_{\tau}\left(\kappa_{\Delta}\right)=E\left[v(c) \mid c \in\left[0, \kappa_{\Delta}\right]\right] \text { for all } c \leq \kappa_{\Delta} .
$$

On the other hand, it is immediate that in the laissez-faire economy the equilibrium payoff is weakly increasing in type (by revealed preference, type $c^{\prime}$ can trade at the same time and price as any type $c<c^{\prime}$ and since $c^{\prime}$ gets a higher payoff flow from holding his asset, his 
payoff from this strategy is at least as high as type's $c$ ). Combining these observations yields:

$$
U_{L F}(c) \leq U_{L F}\left(\kappa_{\Delta}\right)<U_{\tau}\left(\kappa_{\Delta}\right)=U_{\tau}(c) \text { for all } c \leq \kappa_{\Delta}
$$

and that finishes the proof.

Proof of Theorem 2. The optimization problem to find the welfare-maximizing Paretoimproving allocation can be written as:

$$
\begin{array}{rl} 
& \max _{x(c), U(1) \geq 1} \int_{0}^{1} x(c)[v(c)-c] f(c) d c \\
\text { s.t. } & \int_{0}^{1} x(c)\left[v(c)-c-\frac{F(c)}{f(c)}\right] f(c) d c-(U(1)-1)+b \\
0 \leq & \left(U(1)-\int_{c}^{1}(1-x(\tilde{c})) d \tilde{c}\right)-\left(1-\int_{c}^{1}(1-y(\tilde{c})) d \tilde{c}\right) \text { for all } c \\
0 & x(c) \text { weakly decreasing, }
\end{array}
$$

where recall from the body of the paper that $y(c) \equiv e^{-r t(c)}$ is the discount factor related to the time type $c$ trades in the laissez-faire equilibrium.

The first constraint is the usual budget constraint. The second set of constraints is new: they are equivalent to $U(c) \geq U_{L F}(c)$, where we used $U_{L F}(1)=1$ and $U^{\prime}(c)=(1-x(c))$. Re-arranging terms, these constraints simplify to:

$$
0 \leq U(1)-1+\int_{c}^{1}(x(\tilde{c})-y(\tilde{c})) d \tilde{c} \text { for all } c
$$

We refer to these Pareto-improvement constraints as "IR constraints." Note that the IR constraint for type $c$ depends only on the allocations for types $c^{\prime}>c$, changing the allocations for lower types does not affect it.

For part $(i)$ of the theorem, note that for small $c, v(c)-c-\frac{F(c)}{f(c)}>0$. Hence, setting $x(c)=1$ increases the objective function while relaxing the budget constraint and all the IR constraints. Hence, there is a range of types (at the very least those with $v(c)-c-\frac{F(c)}{f(c)}>0$, but possibly more), for which the optimal Pareto-improving mechanism sets $x(c)=1$. 
For part (ii) define

$$
L_{P}(c) \equiv(v(c)-c) f(c)(1+\Lambda)-F(c) \Lambda
$$

where $\Lambda$ is the Lagrange-multiplier on the budget constraint. ${ }^{31}$ Note that $L_{P}(c)$ is strictly decreasing since $\Lambda>0$ and we assumed that $(v(c)-c) f(c)$ is weakly decreasing.

We can re-state the optimization problem as:

$$
\begin{aligned}
& \max _{x(c), \Gamma \geq 0} \int_{0}^{1} x(c) L_{P}(c) d c-\Lambda \Gamma \\
\text { s.t. } & \\
0 \leq & \Gamma+\int_{c}^{1}(x(\tilde{c})-y(\tilde{c})) d \tilde{c} \text { for all } c \\
& x(c) \text { weakly decreasing, }
\end{aligned}
$$

where $\Gamma \equiv U(1)-U_{L F}(1)$.

We claim that the optimal solution to $(22)$ is $\Gamma=0$ and:

$$
x(c)=\left\{\begin{array}{c}
1 \text { for } c \leq c^{*} \\
y(c) \text { for } c>c^{*}
\end{array}\right.
$$

where $c^{*}$ is the largest solution to:

$$
\int_{0}^{c^{*}}\left[v(c)-c-\frac{F(c)}{f(c)}\right] f(c) d c+\int_{c^{*}}^{1} y(c)\left[v(c)-c-\frac{F(c)}{f(c)}\right] f(c) d c+b=0,
$$

so that the budget constraint is binding.

Let $(\Gamma, x(c))$ be the optimal mechanism and without loss of generality assume that $x(c)$ is a càdlàg (right continuous with left limits) function. There are two parts to establish our claim:

Step 1: In the optimal mechanism $\Gamma=0$.

Step 2: Given $\Gamma=0$, in the optimal mechanism $x(c)=y(c)$ for all $c>c^{*}$, and $x(c)=1$ for all $c<c^{*}$, where $c^{*}$ satisfies $L_{P}\left(c^{*}\right)=0$.

\footnotetext{
${ }^{31}$ This is just like $L(c)$ in Theorem 1 except that the multipliers are different.
} 


\section{Step 1:}

Suppose by contradiction that $\Gamma>0$. Since $\Gamma>0$, IR constraints are slack for types close to 1 . Let $c^{\prime}$ be the largest $c$ such that $U(c)=U_{L F}(c)$ (it exists since if we ignored the IR constraints the solution would violate them, and because $U(c)$ and $U_{L F}(c)$ are continuous in $c$ ). Then, $\lim _{c / c^{\prime}}(x(c)-y(c)) \geq 0$ since otherwise the IR constraint would be violated at some $c<c^{\prime}$. Moreover, $x\left(c^{\prime}\right)<y\left(c^{\prime}\right)$ since otherwise the IR constraint would be slack at $c^{\prime}$.

If $\Gamma>0$, we claim that the optimal $x(c)=0$ for $c>c^{\prime}$. Suppose not. Let $c^{\prime \prime} \in\left(c^{\prime}, 1\right)$ be the supremum $c$ such that $x(c)>0$. Then, for $c \in\left(c^{\prime \prime}-\varepsilon^{\prime \prime}, c^{\prime \prime}\right)$ decrease $x(c)$ to 0 and at the same time for $c \in\left(c^{\prime}, c^{\prime}+\varepsilon^{\prime}\right)$ increase $x(c)$ to $y(c)$, picking $\varepsilon^{\prime \prime}$ and $\varepsilon^{\prime}$ in a way that this new allocation $\hat{x}(c)$ satisfies $\int_{c^{\prime}}^{1}[x(c)-\hat{x}(c)] d c=0$ and the IR constraint is still satisfied for all $c>c^{\prime}$. That $\hat{x}(c)$ satisfies IR constraints for all types as well as the monotonicity constraint (recall $\lim _{c / c^{\prime}}(x(c)-y(c)) \geq 0$ ). Finally, since $L_{P}(c)$ is decreasing, $\hat{x}(c)$ improves objective (22) over $x(c)$, a contradiction. So it must be that $x(c)=0$ for all $c \geq c^{\prime}$.

Now reduce $\Gamma$ by $\varepsilon>0$ and construct a new $\hat{x}(c)=(1-\alpha) y(c)$ for $c \geq c^{\prime}$ and $\hat{x}(c)=x(c)$ for $c \leq c^{\prime}$, where $\alpha \in(0,1)$ is a constant such that $\int_{c^{\prime}}^{1}[\hat{x}(c)-x(c)] d c=\varepsilon$, so that for all $c \leq c^{\prime}$ the IR constraints are unaffected and for $c>c^{\prime}$ all IR constraints are still slack. ${ }^{32}$ Since $L_{P}(c)$ is decreasing in $c$, this change improves the objective function by more than

$$
\begin{aligned}
\Lambda \varepsilon+\int_{c^{\prime}}^{1}(\hat{x}(c)-x(c)) L_{P}(c) d c & >\Lambda \varepsilon+\int_{c^{\prime}}^{1}(\hat{x}(c)-x(c)) \underbrace{L_{P}(1)}_{=-\Lambda} d c \\
& =\Lambda \varepsilon-\Lambda \int_{c^{\prime}}^{1}[\hat{x}(c)-x(c)] d c=0
\end{aligned}
$$

so we get a strict improvement since $L_{P}(1)<L_{P}(c)$ for any $c<1$. Therefore, setting $\Gamma>0$ cannot be optimal.

Step 2: Let $x(c)$ be the optimal (càdlàg) solution of (22) with the corresponding Lagrange multiplier $\Lambda$. Let $c^{*}$ be the unique solution to $L_{P}(c)=0$. For types $c<c^{*}$ it is clearly optimal to set $x(c)=1$ since it would relax the IR constraints and make the objective function higher.

Now, suppose that the optimal $x(c) \neq y(c)$ for a range of types above $c^{*}$. Then there

\footnotetext{
${ }^{32}$ Again, since $\lim _{c \uparrow c^{\prime}}(x(c)-y(c)) \geq 0$, this $\hat{x}(c)$ is decreasing.
} 
must exist a range of types above $c^{*}$ for which the IR constraints are slack. Let $c_{1}>c^{*}$ be the supremum of $c$ such that the IR constraints are slack. That implies that $c_{1}$ is the supremum over types such that $x(c)>y(c)$ and for all $c>c_{1}$ it must be that $x(c)=y(c)$ since $\Gamma=0$ and the IR constraints bind. Note that the IR must bind at $c=c^{*}$ since otherwise we could reduce $x(c)$ in the neighborhood of $\left(c^{*}, c^{*}+\varepsilon\right)$ and improve the objective function. ${ }^{33}$ Therefore, there exist constants $c_{4}<c_{3} \leq c_{2}<c_{1}$ such that:

$$
\begin{aligned}
x(c) & >y(c) \text { for all } c \in\left(c_{2}, c_{1}\right) \\
x(c) & =y(c) \text { for all } c \in\left(c_{3}, c_{2}\right) \\
x(c) & <y(c) \text { for all } c \in\left(c_{4}, c_{3}\right) \\
\lim _{c / c_{4}} x(c) & \geq y(c),
\end{aligned}
$$

where we allow for $c_{3}=c_{2}$ and the set $\left(c_{3}, c_{2}\right)$ to be empty. Then we can find $\varepsilon_{1}$ and $\varepsilon_{4}$ (both positive) and adjust the allocation $x(c)$ so that $\hat{x}(c)=y(c)$ for $c \in\left(c_{1}-\varepsilon_{1}, c_{1}\right)$ and for $c \in\left(c_{4}, c_{4}+\varepsilon_{4}\right)$, and $\hat{x}(c)=x(c)$ for all other $c$, where $\varepsilon_{1}$ and $\varepsilon_{4}$ are chosen such that $\int_{c^{\prime}}^{c_{1}}(\hat{x}(c)-y(c)) d c \geq 0$ for all $c^{\prime} \geq c_{4}$ and $\int_{c_{4}}^{c_{1}}(\hat{x}(c)-x(c)) d c=0$ so that the IR constraints for $c<c_{4}$ remain unchanged. This $\hat{x}(c)$ still satisfies monotonicity and IR constraints. Yet, since $L_{P}(c)$ is decreasing, it improves the value functions, a contradiction. The figure below illustrates the constants and the construction, with $c_{2}=c_{3}$ and with the allocation $x(c)$ decreasing discontinuously at $c_{1}$.

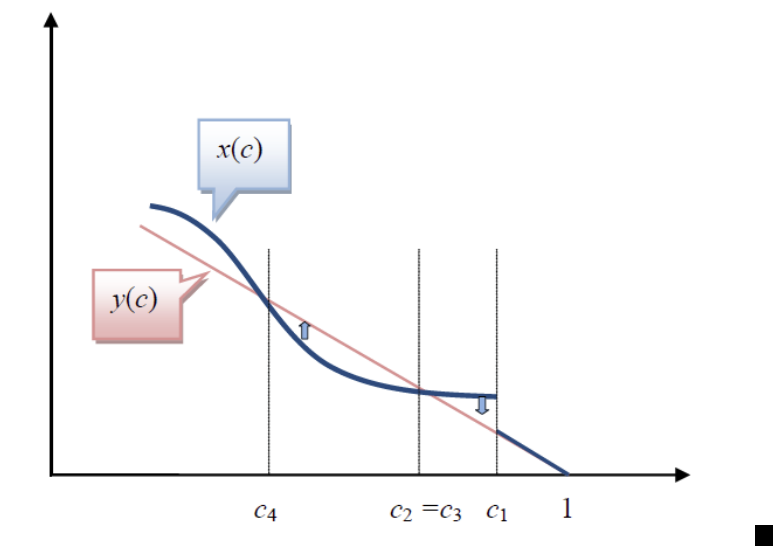

\footnotetext{
${ }^{33}$ To satisfy the monotonicity condition it may require reducing $x(c)$ over a larger range if $x(c)$ is constant over the range $\left(c^{*}, c^{*}+\varepsilon\right)$.
} 
Proof of Proposition 2. Denote $z=e^{-r T}$. Then the surplus can be written as:

$$
S(z)=z\left(\int_{0}^{\sqrt{\frac{4 b}{z}}}\left(\frac{1-c}{2}\right) d c+\int_{\sqrt{\frac{4 b}{z}}}^{1}\left(\frac{1-c}{1-\sqrt{\frac{4 b}{z}}}\right)\left(\frac{1-c}{2}\right) d c\right)=\frac{1}{6} z-\frac{1}{3} b+\frac{1}{3} z \sqrt{\frac{b}{z}} .
$$

So the surplus is increasing in $e^{-r T}$.

Evaluating the surplus at $T=\bar{T}$, so that $b=\frac{1}{4} \frac{(1-z)^{2}}{z}$, for $\bar{T}$ small enough that $\kappa<1$ (so that $\left.e^{-r T} \in\left(\frac{1}{2}, 1\right)\right)$, we get

$$
S(b, \bar{T})=\frac{1}{12 z}\left(4 z-z^{2}-1\right),
$$

which is less than $\frac{1}{6}$ for all $e^{-r T}<1$ (for $z \leq 1$ it is an increasing function and at $z=1$ it is $\left.\frac{1}{6}\right)$.

Proof of Proposition 3. As we explained in the text, in case $r>\lambda$ the equilibrium is described by the differential equation

$$
(r-\lambda)\left(1-k_{t}\right)=\dot{k}_{t}
$$

Using the boundary condition $k_{0}=0$, we get a unique solution:

$$
k_{t}=1-e^{-(r-\lambda) t} .
$$

If the subsidy arrives at time $t$, total surplus is:

$$
\begin{aligned}
S(t \mid \lambda) & =\int_{0}^{t} e^{-r s}\left(v\left(k_{s}\right)-k_{s}\right) \dot{k}_{s} d s+e^{-r t} \int_{k_{t}}^{1}(v(c)-c) d c \\
& =\frac{1}{4} \frac{2(r-\lambda)+r e^{-t(3 r-2 \lambda)}}{3 r-2 \lambda} .
\end{aligned}
$$

Taking expectation over the arrival time:

$$
S(\lambda)=\int_{0}^{\infty} S(t \mid \lambda)\left(\lambda e^{-\lambda t}\right) d t=\frac{1}{4} \frac{2 r-\lambda}{3 r-\lambda},
$$

which is decreasing in $\lambda$.

For the second part, when $\lambda>r$, since upon arrival the government induces the first-best 
surplus, which is $\frac{1}{4}$ in our example, total surplus is

$$
S(\lambda)=\frac{1}{4} \frac{\lambda}{r+\lambda}
$$

\section{References}

[1] Akerlof, George. A. (1970). "The Market for "Lemons": Quality Uncertainty and the Market Mechanism." Quarterly Journal of Economics, 84 (3), pp. 488-500.

[2] Biais, Bruno, Larry Glosten, and Chester Spatt. "Market microstructure: A survey of microfoundations, empirical results, and policy implications." Journal of Financial Markets 8.2 (2005): 217-264

[3] Bolton, Patrick, Tano Santos and Jose A. Scheinkman, 2011. "Outside and Inside Liquidity," The Quarterly Journal of Economics, Oxford University Press, vol. 126(1), pages 259-321

[4] Brusco, Sandro and Hugo Hopenhayn, 2007. "Deregulation with Consensus" Economic Theory, 32, 223-250.

[5] Camargo, Braz and Benjamin Lester (2014). "Trading Dynamics in Decentralized Markets with Adverse Selection." Journal of Economic Theory, 2014, 153, pp. 534-568..

[6] Chiu, Jonathan and Thorsten V. Koeppl (2011) "Trading Dynamics with Adverse Selection and Search: Market Freeze, Intervention and Recovery" Working Paper Bank of Canada.

[7] Daley, Brendan and Brett Green (2012) "Waiting for News in the Market for Lemons." Econometrica 80 (4), 1433-1504

[8] Duffie, Darrell. 2010. "The Failure Mechanics of Dealer Banks." Journal of Economic Perspectives, 24(1): 51-72.

[9] Fernandez, Raquel and Dani Rodrik (1991). "Resistance to Reform: Status Quo Bias in the Presence of Individual- Specific Uncertainty" The American Economic Review, Vol. 81, No. 5, pp. 1146-1155 
[10] Fuchs, William and Andrzej Skrzypacz (2013) "Costs and Benefits of Dynamic Trading in a Lemons Market" Stanford GSB Research Paper Series \# 2133. August 2013.

[11] Fuchs, William, Aniko Öry and Andrzej Skrzypacz (2015) "Transparency and Distressed Sales under Asymmetric Information." Cowles Foundation Discussion Paper No. 1986.

[12] Heider, Florian, Marie Hoerova, and Cornelia Holthausen, 2009. "Liquidity hoarding and interbank market spreads: the role of counterparty risk," Working Paper Series 1126, European Central Bank.

[13] Hörner, Johannes and Nicolas Vieille (2009) "Public vs. Private Offers in the Market for Lemons." Econometrica, 77 (1), pp. 29-69.

[14] Janssen, Maarten C. W. and Santanu Roy (2002) "Dynamic Trading in a Durable Good Market With Asymmetric Information." International Economic Review, 43 (1), pp. 257-282.

[15] Kaya, Ayca and Liu, Qingmin (2012) "Transparency and Price Formation," working paper.

[16] Kremer, Ilan and Andrzej Skrzypacz (2007) "Dynamic Signaling and Market Breakdown." Journal of Economic Theory, 133 (1), pp. 58-82.

[17] Kim, Kyungmin (2012) "Information about Sellers' Past Behavior in the Market for Lemons," working paper.

[18] Mas-Colell, Andreu; Michael D. Whinston and Jerry R. Green (1995). Microeconomic Theory. New York: Oxford University Press.

[19] McCabe, Patrick (2010), "The Cross Section of Money Market Fund Risks and Financial Crises," Federal Reserve Board, Finance and Economics Discussion Series \#2010-51;

[20] Moreno, Diego and John Wooders (2012). "Dynamic Markets for Lemons: Performance, Liquidity, and Policy Intervention," University Carlos III de Madrid Working paper 1226.

[21] Nöldeke, Georg and Eric van Damme (1990) "Signalling in a Dynamic Labour Market." Review of Economic Studies, 57 (1), pp. 1-23.

[22] Philippon, Thomas and Skreta, Vasiliki (2012) "Optimal Interventions in Markets with Adverse Selection", American Economic Review, 102 (1), pp. 1-28. 
[23] Samuelson, William (1984) "Bargaining under Asymmetric Information." Econometrica, 52 (4), pp. 995-1005.

[24] Sobel, Joel (1991) "Durable Goods Monopoly with Entry of New Consumers" Econometrica, Vol. 59, No.5 1455-1485.

[25] Spence, Michael (1973) "Job Market Signaling.", Quarterly. Journal of Economics, 87, pp. $355-374$.

[26] Swinkels, Jeroen M. (1992) "Educational Signalling With Preemptive Offers." Review of Economic Studies, 66 (4), pp. 949-970.

[27] Tirole, Jean (2012) "Overcoming Adverse Selection: How Public Intervention Can Restore Market Functioning." American Economic Review, 102 pp. 19-59. 